\title{
Evidence for the Associated Production of a Single Top Quark and a Photon in Proton-Proton Collisions at $\sqrt{s}=13 \mathrm{TeV}$
}

\author{
A. M. Sirunyan et al. \\ (CMS Collaboration)
}

(Received 8 August 2018; published 29 November 2018)

\begin{abstract}
The first evidence of events consistent with the production of a single top quark in association with a photon is reported. The analysis is based on proton-proton collisions at $\sqrt{s}=13 \mathrm{TeV}$ and recorded by the CMS experiment in 2016, corresponding to an integrated luminosity of $35.9 \mathrm{fb}^{-1}$. Events are selected by requiring the presence of a muon $(\mu)$, a photon $(\gamma)$, an imbalance in transverse momentum from an undetected neutrino $(\nu)$, and at least two jets $(j)$ of which exactly one is identified as associated with the hadronization of a $b$ quark. A multivariate discriminant based on topological and kinematic event properties is employed to separate signal from background processes. An excess above the backgroundonly hypothesis is observed, with a significance of 4.4 standard deviations. A fiducial cross section is measured for isolated photons with transverse momentum greater than $25 \mathrm{GeV}$ in the central region of the detector. The measured product of the cross section and branching fraction is $\sigma(p p \rightarrow t \gamma \mathrm{j}) \mathcal{B}(t \rightarrow \mu \nu b)=$ $115 \pm 17$ (stat) \pm 30 (syst) fb, which is consistent with the standard model prediction.
\end{abstract}

DOI: 10.1103/PhysRevLett.121.221802

The study of top quark production in association with a photon is an important test of the standard model (SM) description of top quark interactions with gauge bosons, and plays an important role in the search for physics beyond the SM. The cross section for single top quark production in association with a photon is sensitive to the top quark charge, and to its electric and magnetic dipole moments $[1,2]$.

The cross section for the production of a top quarkantiquark pair and an associated photon $(t \bar{t}+\gamma)$ has been measured by the CDF Collaboration at the Fermilab Tevatron in proton-antiproton collisions at $\sqrt{s}=1.96 \mathrm{TeV}$ [3], and by the ATLAS and CMS Collaborations in protonproton $(p p)$ collisions at the CERN LHC at $8 \mathrm{TeV}[4,5]$. All measurements are consistent with SM predictions.

The SM predicts three different mechanisms for the production of a single top quark in association with a photon at the LHC: $t$-channel, $s$-channel, and $t W$ production, where the largest contribution comes from the $t$ channel. This Letter presents first evidence for single top quark production in association with a photon in the $t$ channel. The data were collected in 2016 by the CMS experiment at $\sqrt{s}=13 \mathrm{TeV}$ and correspond to an integrated luminosity of $35.9 \mathrm{fb}^{-1}$. One of the distinctive

\footnotetext{
*Full author list given at the end of the Letter.

Published by the American Physical Society under the terms of the Creative Commons Attribution 4.0 International license. Further distribution of this work must maintain attribution to the author(s) and the published article's title, journal citation, and DOI. Funded by SCOAP ${ }^{3}$.
}

signatures of the signal is the presence of a forward light-flavor energetic jet in the final state that arises from the spacelike nature of the $W$ boson propagator. The search targets events with a top quark, a photon, and at least one additional light-flavor jet, $(t \gamma \mathrm{j})$ where the top quark decays into a $W$ boson and a $b$ quark, followed by the $W$ boson decay into a muon and a neutrino. Figure 1 shows representative Feynman diagrams for the process $t \gamma \mathrm{j}$ including the leptonic decay of the $W$ boson produced in the top quark decay.

This analysis focuses on the muon decay channel since it enables a good signal selection efficiency with low background contamination. The final state includes possible contributions from $W \rightarrow \tau \nu_{\tau}$ where the $\tau$ lepton decays to $\mu \nu_{\mu} \nu_{\tau}$. The kinematic and topological properties of the signal are exploited through a multivariate technique to discriminate against the background processes. The background can be divided into two types: events which contain a genuine photon, such as $t \bar{t}+\gamma, W \gamma+$ jets, and $Z \gamma+$ jets and events in which the photon candidate arises from a misidentified jet, such as $t \bar{t}, W+$ jets, and $Z+$ jets processes.

The central feature of the CMS apparatus is a superconducting solenoid of $6 \mathrm{~m}$ internal diameter, providing a magnetic field of $3.8 \mathrm{~T}$. A silicon pixel and strip tracker, a lead tungstate crystal electromagnetic calorimeter (ECAL), and a brass and scintillator hadron calorimeter, each composed of a barrel and two end cap sections, are contained within the superconducting solenoid volume. Extensive forward calorimetry complements the coverage provided by the barrel and end cap detectors. Muons are detected in gas-ionization detectors embedded in the steel 

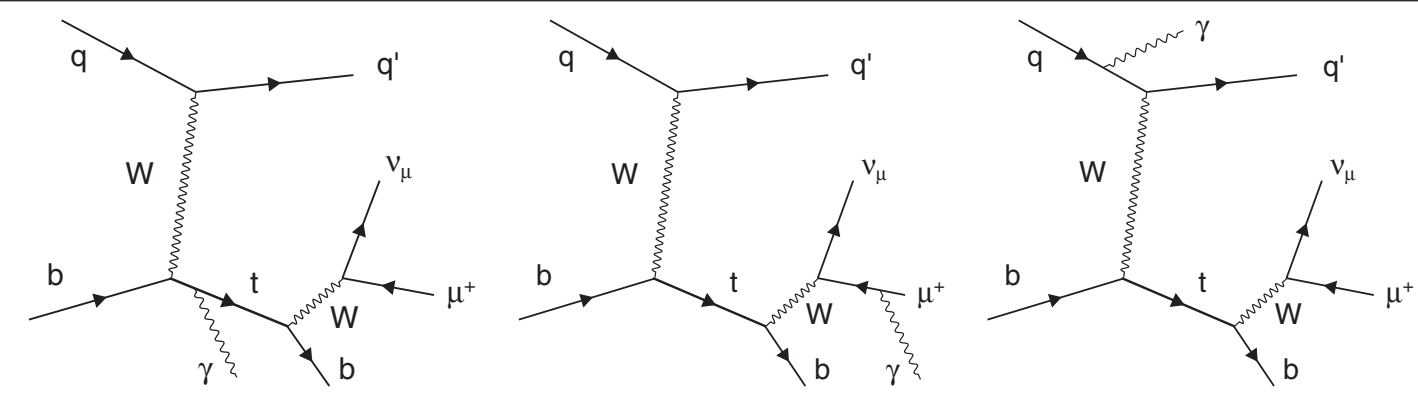

FIG. 1. Representative $t$-channel Feynman diagrams for single top quark production in association with a photon, including the leptonic decay of the $W$ boson produced in the top quark decay.

flux-return yoke outside the solenoid. Online event selection is accomplished via the two-tiered CMS trigger system [6]. A more detailed description of the CMS detector, together with a definition of the coordinate system and kinematic variables used in this analysis, can be found in Ref. [7].

Simulated samples for the $t \gamma \mathrm{j}$ signal are generated at next-to-leading order (NLO) using the MADGRAPH5 AMC@NLO v 2.2.2 event generator [8], with a minimum transverse momentum requirement of $p_{\mathrm{T}}>10 \mathrm{GeV}$ and a pseudorapidity requirement of $|\eta|<2.6$ for the associated photon. The angular separation between the photon and all other particles is required to be $\Delta R>0.05$, where $\sqrt{(\Delta \eta)^{2}+(\Delta \phi)^{2}}$ and $\phi$ is the azimuthal angle in radians. The NNPDF3.0 [9] parton distribution functions (PDFs) are used and the top quark mass is set to $172.5 \mathrm{GeV}$. After these requirements, an inclusive cross section of $2.95 \pm$ $0.13 \pm 0.03 \mathrm{pb}$ is obtained, where the first uncertainty is associated with the renormalization and factorization scales and the second uncertainty with the PDFs. These uncertainties are derived using the SYSCALC program [10].

Samples of simulated events for the production of $t \bar{t}+\gamma$, $W+$ jets, $W \gamma+$ jets, Drell-Yan events, $Z \gamma+$ jets, and diboson $+\gamma$ are generated at NLO using MADGRAPH5_ AMC@NLO. Single top quark events in $t, s$, and $t W$ channels, as well as $t \bar{t}$ events, are generated with the NLO POWHEG v2 event generator [11-14]. The overlaps between the $t \gamma \mathrm{j}$ signal sample and the $t j$ inclusive sample, and between $V \gamma+$ jets and $V+$ jets samples are removed, where $V$ is a $W$ or $Z$ boson.

Showering and hadronization for all of the simulated samples are implemented with PYTHIA v8.212 $[15,16]$ with the underlying event tune CUETP8M1 [17]. To match the generated events with PYTHIA v8.212 parton shower, the FxFx [18] and the MLM [19] prescriptions are used.

The CMS detector response for all simulated samples is modeled using GEANT4 v9.4 [20]. The simulated samples include the presence of additional $p p$ interactions in the same or neighboring bunch crossings (pileup). The distribution of pileup events in simulation is weighted to match that observed in data.

The particle-flow (PF) algorithm [21] is used for the identification and reconstruction of individual particles in an event using a combination of information from all subdetectors. It identifies each reconstructed particle as a muon, an electron, a photon, a neutral or a charged hadron. Data samples are selected based on a single-muon trigger, requiring a muon with $p_{t}>24 \mathrm{GeV}$ and $|\eta|<2$.4. Events are required to have a well-reconstructed primary vertex which is identified as the one with the largest value of summed particle $p_{\mathrm{T}}^{2}$ [22]. The simulated events are weighted in order to reproduce the reconstruction and trigger efficiencies in data.

Jets are reconstructed from the PF candidates using the anti- $k_{\mathrm{T}}$ algorithm [23] with a distance parameter $R=0.4$, implemented in the FASTJET package [24]. Corrections are applied to the jet energy to subtract the contribution from pileup interactions. Jets are tagged as associated to the hadronization of $b$ quarks ( $b$ jets) using secondary vertex algorithm, which combines the secondary vertex and trackbased lifetime information [25]. The working point chosen for the algorithm, corresponds to a $b$ tagging efficiency of $70 \%$, and misidentification rates of $1 \%$ and $15 \%$ for light quark jets and $c$ jets, respectively [25]. Events are required to have at least two jets with $p_{\mathrm{T}}>40 \mathrm{GeV}$, one of which must be $b$-tagged with $|\eta|<2.5$ and another must lie within the range $|\eta|<4.7$.

Events are required to contain exactly one isolated muon with $p_{\mathrm{T}}>26 \mathrm{GeV}$ and $|\eta|<2.4$. The isolation is calculated from the reconstructed charged and neutral $\mathrm{PF}$ candidates and is corrected for pileup effects [26]. In order to reduce the contribution of background processes with multiple leptons in the final state, events containing additional muon candidates satisfying loose selection criteria or containing electron candidates are rejected [26].

Photon candidates are built from clusters of high-energy deposits in the ECAL. Photon identification depends on isolation and shower shape variables which reflect the energy dispersion in $\eta$, and is described in detail in Ref. [27]. The effects of pileup on the isolation variables are accounted for [28].

A conversion-safe electron veto algorithm [27] is used to reject electrons. It discards events containing a track with an energy deposit in the innermost layer of the pixel detector which is not connected to a reconstructed conversion vertex from a photon cluster in the ECAL. Events 
are required to have exactly one isolated photon candidate with $p_{\mathrm{T}}>25 \mathrm{GeV}$ and $|\eta|<1.44$.

The missing transverse momentum $p_{\mathrm{T}}^{\text {miss }}$ is defined as the negative vector sum of the momenta of all reconstructed $\mathrm{PF}$ objects in the transverse plane. The magnitude of $p_{\mathrm{T}}^{\text {miss }}$ is required to be larger than $30 \mathrm{GeV}$.

In order to select well-isolated objects, the photon is required to be separated from the $b$ jet, the light-flavor jet, and any muon candidates by $\Delta R(X, \gamma)>0.5$, where $X$ stands for $\mu, b$ jet, or light-flavor jet. The angular separation between the muon candidate and jets is required to satisfy $\Delta R(\mu$, jets $)>0.3$. The signal region is defined as the region where all the requirements described above are satisfied.

The top quark is reconstructed using the muon, the $b$ jet candidate, and $p_{T}^{\text {miss }}$ where the transverse momentum of the neutrino, which escapes detection, is assumed to be equal to $p_{T}^{\text {miss }}$. The longitudinal component of the neutrino momentum is obtained by constraining the invariant mass of the muon and the neutrino to the $W$ boson mass, resulting in a quadratic equation. When the two solutions are real, the one with smaller absolute value is taken, while when the solutions are complex the real part is taken [29]. The top quark momentum is reconstructed by combining the momenta of the $b$ jet and of the reconstructed $W$ boson.

The backgrounds from $W \gamma+$ jets, $Z \gamma+$ jets, diboson $+\gamma, s$, and $t W$ channel single top quark with a photon production are estimated using simulation. The contribution of the misidentified photon background is estimated from the measurement of the $p_{T}$-dependent probability for a jet to be reconstructed as a photon using the method described in Refs. [30,31]. The misidentification probability is calculated based on the near independence of the shower shape and photon isolation variables, which permits the factorization of the misidentification probability and hence its estimation outside the signal region.

Several sources of uncertainties are considered in this estimation. The first comes from the definition of the sideband region and is due to possible correlations between the photon shower shape and photon isolation, that are assumed to be independent. The second source is due to the small contribution of genuine photons in the sideband region, which is estimated from simulation. The last source of uncertainty arises from the limited number of events available in the various photon $p_{T}$ bins considered in the estimation of the background from misidentified jets.

The uncertainties in the lepton trigger, reconstruction, and identification efficiencies are evaluated using DrellYan events $[26,32,33]$. The uncertainties from the photon selection efficiency and photon energy scale are obtained using $Z \rightarrow e^{+}+e^{-}$events. The photon energy scale uncertainty is found to be $1 \%[34,35]$. The uncertainty from pileup modeling is evaluated by varying the total $p p$ inelastic cross section by $4.6 \%$ [36] and reweighting the simulation accordingly. The uncertainties in the jet energy resolution and jet energy scale (JES) $[37,38]$, and $p_{T}^{\text {miss }}$ are
TABLE I. Event yields after the event selection in data and for each SM contribution. The expected yields are presented with their statistical and systematic uncertainties combined.

\begin{tabular}{lc}
\hline \hline Process & Event yield \\
\hline$t \bar{t}+\gamma$ & $1401 \pm 131$ \\
$W \gamma+$ jets & $329 \pm 78$ \\
$Z \gamma+$ jets & $232 \pm 55$ \\
Misidentified photon & $374 \pm 74$ \\
$t \gamma(s$ and $t W$ channel $)$ & $57 \pm 8$ \\
VV $\gamma$ & $8 \pm 3$ \\
Total background & $2401 \pm 178$ \\
Expected signal & $154 \pm 24$ \\
Total SM prediction & $2555 \pm 180$ \\
Data & 2535 \\
\hline \hline
\end{tabular}

also considered. The $b$ jet identification and misidentification corrections are applied as a function of the $p_{T}$ and $\eta$ of the jet [25]. The systematic uncertainty in the integrated luminosity is estimated to be $2.5 \%$ [39]. The uncertainties in the $W \gamma+$ jets, $Z \gamma+$ jets, $\mathrm{VV} \gamma$, and single top quark contributions are based on the CMS measurements from Refs. [31,40,41,42], respectively. Several sources of uncertainties in the signal modeling are taken into account. The uncertainty from the variations of renormalization and factorization scales is estimated by comparing the simulated samples with scales halved and doubled with respect to the initial value. The uncertainty from the PDF choice is evaluated using the replicas of the NNPDF3.0 PDF set. The uncertainty from parton shower modeling and hadronization is obtained by comparing the results derived from PYTHIA v8.212 and HERWIG++ v2.3 [43].

After the selection, the total number of observed events is 2535 of which $2401 \pm 178$ events are expected from the SM background in absence of $t \gamma \mathrm{j}$ signal, where the uncertainty includes both the statistical and systematic components. The expected number of signal events from the $\mathrm{SM}$ is $154 \pm 24$. The dominant background is $t \bar{t}+\gamma$, and amounts to $55 \%$ of the total background yield. Event yields in data and simulation for all background processes are presented in Table I where the $t \bar{t}+\gamma$ yield and its uncertainty are predicted from simulation.

The SM prediction for the signal is much smaller than the background, so it is important to optimize the separation between signal and background events. A multivariate classification approach, based on a boosted decision tree (BDT) [44,45], is used. The BDT input variables in decreasing order of importance are (i) $\eta$ of the light-flavor jet, (ii) $\cos \theta$, which is the cosine of the angle between the muon candidate and the light-flavor jet in the top quark rest frame, (iii) $\eta$ of the muon candidate, (iv) $\Delta R$ (light-flavor jet, $\gamma$ ), (v) reconstructed top quark mass $m_{\mu \nu b}$, (vi) jet multiplicity, (vii) transverse mass of the reconstructed $W$ boson, and (viii) muon charge. The distributions of some of the most important BDT input variables for data and SM 

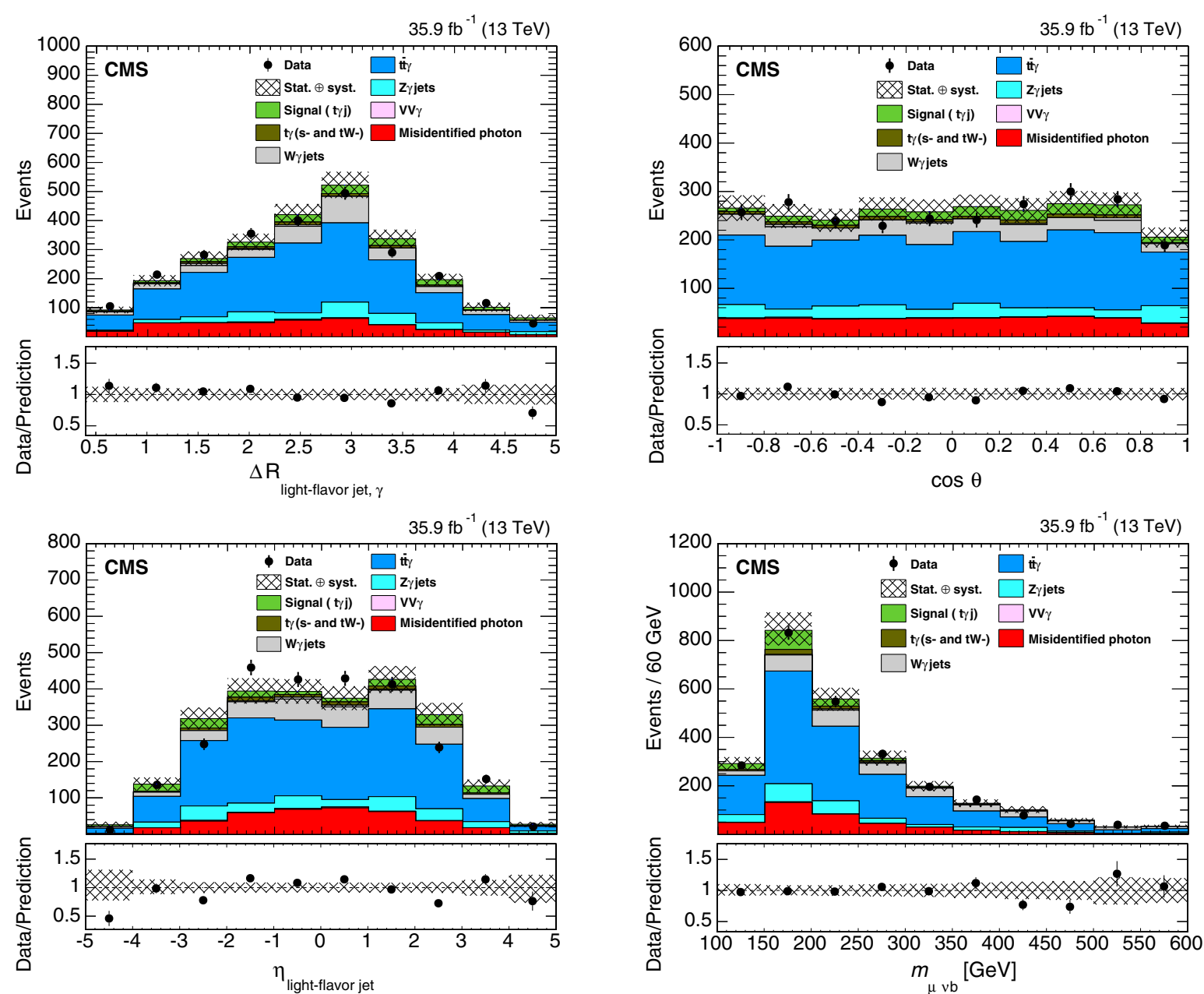

FIG. 2. Distributions of some of the input variables to the BDT: $\Delta R$ (light jet, $\gamma$ ) (upper left), $\cos \theta$ (upper right), $\eta$ of the light-flavor jet (lower left), and $m_{\mu \nu b}$ (lower right) after the final event selection in data (points), and the SM prediction (filled histograms). The hatched band shows the statistical and systematic uncertainties in the estimated signal and background yields, and the vertical bars on the points represent the statistical uncertainties of the data. The ratios of the data to the SM predictions are shown in the bottom panels.

predictions after the event selection are presented in Fig. 2, where the hatched band indicates the overall uncertainty on the SM predictions. It includes a sizable statistical component besides the systematic uncertainties, which are all propagated to the final results.

The BDT is trained to distinguish signal from background and provides a single discriminant value for every event. The BDT output distribution in data is parametrized as

$$
\begin{aligned}
F(x)= & C_{\text {sig }} S_{\text {sig }}(x)+C_{t \overline{t \gamma}} S_{t \bar{t} \gamma}(x)+C_{W \gamma \mathrm{j}} S_{W \gamma \mathrm{j}}(x) \\
& +C_{Z \gamma \mathrm{j}} S_{Z \gamma \mathrm{j}}(x)+C_{\text {misid }} S_{\text {misid }}(x)+C_{\mathrm{B}} S_{\mathrm{B}}(x),
\end{aligned}
$$

where $x$ is the BDT output, $S_{\text {sig }}(x), S_{W \gamma \mathrm{j}}(x), S_{Z \gamma \mathrm{j}}(x)$, $S_{\text {misid }}(x)$, and $S_{\mathrm{B}}(x)$ are the normalized distributions (templates) for the signal, $t \bar{t}+\gamma, W \gamma+$ jets, $Z \gamma+$ jets, the misidentified photon background, and the sum of all other backgrounds which includes single top quark production and $\mathrm{VV} \gamma$, respectively. The quantities $C_{\mathrm{sig}}, C_{t \bar{\gamma} \gamma}$,
$C_{W \gamma \mathrm{j}}, C_{Z \gamma \mathrm{j}}, C_{\text {misid }}$, and $C_{\mathrm{B}}$ are the corresponding normalizations from which $C_{\text {sig }}$ and $C_{t \bar{t} \gamma}$ are left free in the fit, while the other terms are constrained by their associated uncertainties. The different templates, $S(x)$, are taken from simulation except for $t \bar{t}+\gamma$ and misidentified photons. The distribution $S_{\text {misid }}(x)$ is obtained with the method described above, calculating the yield as a function of BDT output. The template $S_{t \bar{t} \gamma}(x)$ is estimated from data using a control region defined by requiring exactly two $b$-tagged jets, while keeping all other selection criteria the same as for the signal region. The requirement of two $b$-tagged jets ensures a high contribution from $t \bar{t}+\gamma$, while suppressing the contributions from all other processes. The uncertainty in the difference of shape between events with two $b$-tagged and one $b$-tagged jet is calculated using simulation and accounted for as a systematic uncertainty. In addition, $S_{W \gamma \mathrm{j}}(x)$ is validated in data, in a control region enriched in $W \gamma+$ jets events, via the substitution of the signal $b$ jet requirement by a $b$ jet veto. 


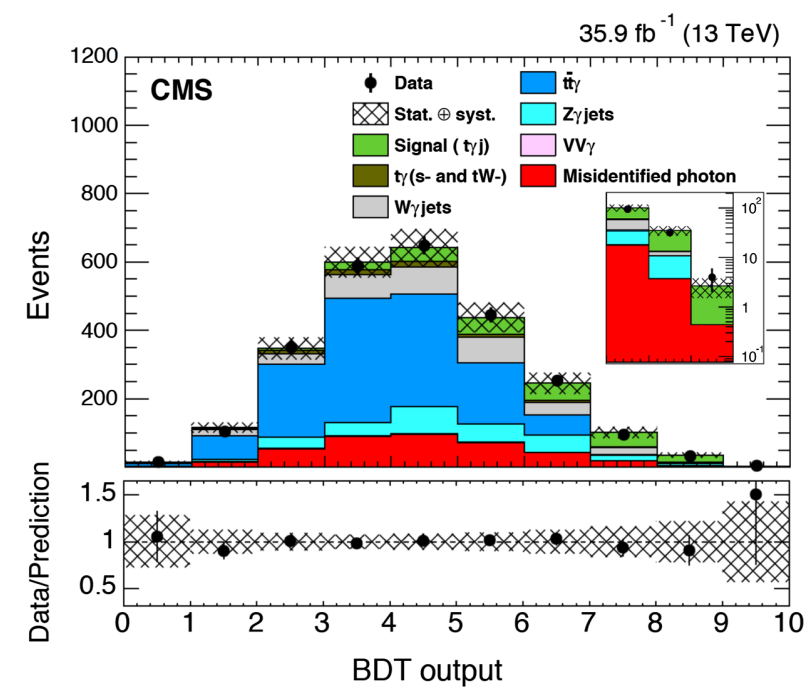

FIG. 3. The BDT output distribution for data and SM predictions after performing the fit. The inset presents a closeup of the last three bins plotted on log scale. The hatched band shows the statistical and systematic uncertainties in the estimated signal and background yields, and the vertical bars on the points represent the statistical uncertainties of the data. The ratio of the data to the SM prediction is shown in the bottom panel.

In order to extract the signal cross section and $t \bar{t}+\gamma$ background normalization, a simultaneous binned likelihood fit is performed on the BDT distribution in the signal region and the $t \bar{t}+\gamma$ control region. Including events from the $t \bar{t}+\gamma$ control region in the fit is useful to constrain the $t \bar{t}+\gamma$ background normalization. Each source of systematic uncertainty is included as a nuisance parameter in the likelihood function. The normalizations of backgrounds except for $t \bar{t}+\gamma$ are left free to vary within the systematic uncertainties. A profile likelihood ratio test statistic is constructed by generating pseudodata for the background-only and for the signal-plus-background hypotheses. The BDT output distribution for data and SM prediction after the fit is shown in Fig. 3.

All of the systematic uncertainties affect both the normalization of backgrounds and shape of the BDT discriminant, except those associated with the integrated luminosity, photon energy scale, $p_{T}^{\text {miss }}$, and background rates that only affect the normalization. The shape uncertainties have Gaussian constraints, while rate uncertainties have lognormal forms. The main systematic uncertainties in the signal cross section arise from the JES, signal modeling, normalization of $Z \gamma+$ jets, and $b$ tagging and mistagging rates, and amount to $12 \%, 9 \%, 8 \%$, and $7 \%$, respectively. The impact of the uncertainty from each source is calculated by performing the fit with all other nuisance parameters fixed to their fitted values. The number of signal and $t \bar{t}+\gamma$ events after the fit are $220 \pm 63$ and $1221 \pm 121$, which both agree with the expected yields within the uncertainties.

An excess of events above the expected background is observed at a $p$ value [46] of $4.27 \times 10^{-6}$, which corresponds to a significance of 4.4 standard deviations. The median expected significance is 3.0, and the $68 \%$ and $95 \%$ confidence level ranges for the expected significance are $[1.5,4.0]$ and $[0,8.7]$, respectively. A fiducial product of the cross section and branching fraction of $\sigma(p p \rightarrow t \gamma \mathrm{j}) \mathcal{B}(t \rightarrow \mu \nu b)=115 \pm 17$ (stat) \pm 30 (syst) fb is measured in the phase space defined by the photon transverse momentum $p_{T, \gamma}>25 \mathrm{GeV},\left|\eta_{\gamma}\right|<1.44$, and $\Delta R(X, \gamma)>0.5$, where $X$ stands for $\mu, b$ jet, light-flavor jet. The expected SM product of the cross section and branching fraction within this fiducial phase space is $81 \pm 4 \mathrm{fb}$, in agreement with the measurement. This is the first experimental evidence for single top quark production in association with a photon.

We congratulate our colleagues in the CERN accelerator departments for the excellent performance of the LHC and thank the technical and administrative staffs at CERN and at other CMS institutes for their contributions to the success of the CMS effort. In addition, we gratefully acknowledge the computing centres and personnel of the Worldwide LHC Computing Grid for delivering so effectively the computing infrastructure essential to our analyses. Finally, we acknowledge the enduring support for the construction and operation of the LHC and the CMS detector provided by the following funding agencies: BMWFW and FWF (Austria); FNRS and FWO (Belgium); CNPq, CAPES, FAPERJ, and FAPESP (Brazil); MES (Bulgaria); CERN; CAS, MoST, and NSFC (China); COLCIENCIAS (Colombia); MSES and CSF (Croatia); RPF (Cyprus); SENESCYT (Ecuador); MoER, ERC IUT, and ERDF (Estonia); Academy of Finland, MEC, and HIP (Finland); CEA and CNRS/IN2P3 (France); BMBF, DFG, and HGF (Germany); GSRT (Greece); OTKA and NIH (Hungary); DAE and DST (India); IPM (Iran); SFI (Ireland); INFN (Italy); MSIP and NRF (Republic of Korea); LAS (Lithuania); MOE and UM (Malaysia); BUAP, CINVESTAV, CONACYT, LNS, SEP, and UASLP-FAI (Mexico); MBIE (New Zealand); PAEC (Pakistan); MSHE and NSC (Poland); FCT (Portugal); JINR (Dubna); MON, RosAtom, RAS, RFBR and RAEP (Russia); MESTD (Serbia); SEIDI, CPAN, PCTI and FEDER (Spain); Swiss Funding Agencies (Switzerland); MST (Taipei); ThEPCenter, IPST, STAR, and NSTDA (Thailand); TUBITAK and TAEK (Turkey); NASU and SFFR (Ukraine); STFC (United Kingdom); DOE and NSF (USA). Individuals have received support from INSF (Iran).

[1] M. Fael and T. Gehrmann, Probing top quark electromagnetic dipole moments in single-top-plus-photon production, Phys. Rev. D 88, 033003 (2013).

[2] S. M. Etesami, S. Khatibi, and M. M. Najafabadi, Measuring anomalous $W W \gamma$ and $t \bar{t} \gamma$ couplings using top $+\gamma$ production at the LHC, Eur. Phys. J. C 76, 533 (2016). 
[3] T. Aaltonen et al. (CDF Collaboration), Evidence for $t \bar{t} \gamma$ production and measurement of $\sigma_{t \bar{t} \gamma} / \sigma_{t \bar{t}}$, Phys. Rev. D 84, 031104 (2011).

[4] ATLAS Collaboration, Measurement of the $t \bar{t} \gamma$ production cross section in proton-proton collisions at $\sqrt{s}=8 \mathrm{TeV}$ with the ATLAS detector, J. High Energy Phys. 11 (2017) 086.

[5] CMS Collaboration, Measurement of the semileptonic $t \bar{t}+\gamma$ production cross section in $p p$ collisions at $\sqrt{s}=8 \mathrm{TeV}$, J. High Energy Phys. 10 (2017) 006.

[6] CMS Collaboration, The CMS trigger system, J. Instrum. 12, P01020 (2017).

[7] CMS Collaboration, The CMS experiment at the CERN LHC, J. Instrum. 3, S08004 (2008).

[8] J. Alwall, R. Frederix, S. Frixione, V. Hirschi, F. Maltoni, O. Mattelaer, H. S. Shao, T. Stelzer, P. Torrielli, and M. Zaro, The automated computation of tree-level and next-toleading order differential cross sections, and their matching to parton shower simulations, J. High Energy Phys. 07 (2014) 079.

[9] R. D. Ball et al. (NNPDF Collaboration), Parton distributions for the LHC Run II, J. High Energy Phys. 04 (2015) 040 .

[10] A. Kalogeropoulos and J. Alwall, The SysCALC code: A tool to derive theoretical systematic uncertainties, arXiv: 1801.08401 .

[11] P. Nason, A new method for combining NLO QCD with shower Monte Carlo algorithms, J. High Energy Phys. 11 (2004) 040.

[12] S. Frixione, P. Nason, and C. Oleari, Matching NLO QCD computations with parton shower simulations: The POWHEG method, J. High Energy Phys. 11 (2007) 070 (2007).

[13] S. Alioli, P. Nason, C. Oleari, and E. Re, A general framework for implementing NLO calculations in shower Monte Carlo programs: The Powheg Box, J. High Energy Phys. 06 (2010) 043.

[14] J. M. Campbell, R. Keith Ellis, P. Nason, and E. Re, Toppair production and decay at NLO matched with parton showers, J. High Energy Phys. 04 (2015) 114.

[15] T. Sjöstrand, S. Mrenna, and P.Z. Skands, PYTHIA 6.4 physics and manual, J. High Energy Phys. 05 (2006) 026.

[16] T. Sjöstrand, S. Mrenna, and P.Z. Skands, A brief introduction to PYthia 8.1, Comput. Phys. Commun. 178, 852 (2008).

[17] CMS Collaboration, Event generator tunes obtained from underlying event and multiparton scattering measurements, Eur. Phys. J. C 76, 155 (2016).

[18] J. Alwall, S. Höche, F. Krauss, N. Lavesson, L. Lönnblad, F. Maltoni, M. L. Mangano, M. Moretti, C. G. Papadopoulos, F. Piccinini, S. Schumann, M. Treccani, J. Winter, and M. Worek, Comparative study of various algorithms for the merging of parton showers and matrix elements in hadronic collisions, Eur. Phys. J. C 53, 473 (2008).

[19] R. Frederix and S. Frixione, Merging meets matching in MC@NLO, J. High Energy Phys. 12 (2012) 061.

[20] S. Agostinelli et al. (GEANT4 Collaboration), GEANT4: A simulation toolkit, Nucl. Instrum. Methods Phys. Res., Sect. A 506, 250 (2003).
[21] CMS Collaboration, Particle-flow reconstruction and global event description with the CMS detector, J. Instrum. 12, P10003 (2017).

[22] CMS Collaboration, Description and performance of track and primary-vertex reconstruction with the CMS tracker, J. Instrum. 9, P10009 (2014).

[23] M. Cacciari, G. P. Salam, and G. Soyez, The anti- $k_{\mathrm{T}}$ jet clustering algorithm, J. High Energy Phys. 04 (2008) 063.

[24] M. Cacciari, G. P. Salam, and G. Soyez, FAstJet user manual, Eur. Phys. J. C 72, 1896 (2012).

[25] CMS Collaboration, Identification of heavy-flavour jets with the CMS detector in pp collisions at $13 \mathrm{TeV}$, J. Instrum. 13, P05011 (2018).

[26] CMS Collaboration, Performance of the CMS muon detector and muon reconstruction with proton-proton collisions at $\sqrt{s}=13 \mathrm{TeV}$, J. Instrum. 13, P06015 (2018).

[27] CMS Collaboration, Performance of photon reconstruction and identification with the CMS detector in proton-proton collisions at $\sqrt{s}=8 \mathrm{TeV}$, J. Instrum. 10, P08010 (2015).

[28] M. Cacciari and G. P. Salam, Pileup subtraction using jet areas, Phys. Lett. B 659, 119 (2008).

[29] CMS Collaboration, Measurement of the $t$-Channel Single Top Quark Production Cross Section in $p p$ Collisions at $\sqrt{s}=7 \mathrm{TeV}$, Phys. Rev. Lett. 107, 091802 (2011).

[30] CMS Collaboration, Measurement of $W \gamma$ and $Z \gamma$ production in $p p$ collisions at $\sqrt{s}=7 \mathrm{TeV}$, Phys. Lett. B 701, 535 (2011).

[31] CMS Collaboration, Measurement of electroweak-induced production of $W \gamma$ with two jets in $p p$ collisions at $\sqrt{s}=$ $8 \mathrm{TeV}$ and constraints on anomalous quartic gauge couplings, J. High Energy Phys. 06 (2017) 106.

[32] CMS Collaboration, Measurement of Inclusive $W$ and $Z$ Boson Production Cross Sections in $p p$ Collisions at $\sqrt{s}=8 \mathrm{TeV}$, Phys. Rev. Lett. 112, 191802 (2014).

[33] CMS Collaboration, Performance of electron reconstruction and selection with the CMS detector in proton-proton collisions at $\sqrt{s}=8 \mathrm{TeV}$, J. Instrum. 10, P06005 (2015).

[34] CMS Collaboration, Measurement of the $W \gamma$ and $Z \gamma$ inclusive cross sections in $p p$ collisions at $\sqrt{s}=7 \mathrm{TeV}$ and limits on anomalous triple gauge boson couplings, Phys. Rev. D 89, 092005 (2014).

[35] CMS Collaboration, Search for high-mass $Z \gamma$ resonances in proton-proton collisions at $\sqrt{s}=8$ and $13 \mathrm{TeV}$ using jet substructure techniques, Phys. Lett. B 772, 363 (2017).

[36] ATLAS Collaboration, Measurement of the Inelastic Proton-Proton Cross Section at $\sqrt{s}=13 \mathrm{TeV}$ with the ATLAS Detector at the LHC, Phys. Rev. Lett. 117, 182002 (2016).

[37] CMS Collaboration, Jet algorithms performance in $13 \mathrm{TeV}$ data, CMS Physics Analysis Summary, CERN, Report No. CMS-PAS-JME-16-003, 2017, https://cds.cern .ch/record/2256875.

[38] CMS Collaboration, Jet energy scale and resolution in the CMS experiment in pp collisions at $8 \mathrm{TeV}$, J. Instrum. 12, P02014 (2017).

[39] CMS Collaboration, CMS luminosity measurements for the 2016 data taking period, CMS Physics Analysis Summary Report No. CMS-PAS-LUM-17-001, 2017, https://cds.cern .ch/record/2257069.

[40] CMS Collaboration, Measurement of the cross section for electroweak production of $Z \gamma$ in association with two jets and constraints on anomalous quartic gauge couplings in 
proton-proton collisions at $\sqrt{s}=8 \mathrm{TeV}$, Phys. Lett. B 770, 380 (2017).

[41] CMS Collaboration, Measurement of the $W^{+} W^{-}$cross section in $p p$ collisions at $\sqrt{s}=8 \mathrm{TeV}$ and limits on anomalous gauge couplings, Eur. Phys. J. C 76, 401 (2016).

[42] CMS Collaboration, Cross section measurement of $t$-channel single top quark production in $p p$ collisions at $\sqrt{s}=13 \mathrm{TeV}$, Phys. Lett. B 772, 752 (2017).

[43] M. Bähr et al., HERWIG++ physics and manual, Eur. Phys. J. C 58, 639 (2008).
[44] H.-J. Yang, B. P. Roe, and J. Zhu, Studies of boosted decision trees for MiniBooNE particle identification, Nucl. Instrum. Methods Phys. Res., Sect. A 555, 370 (2005).

[45] H. Voss, A. Höcker, J. Stelzer, and F. Tegenfeldt, TMVA, the toolkit for multivariate data analysis with ROOT, arXiv: physics/0703039.

[46] Statistical issues for LHC physics, Proceedings of the Workshop, PHYSTAT-LHC, Geneva, Switzerland, 2007, edited by A. de Roeck, L. Lyons, and H. Prosper (CERN, Geneva, Switzerland, 2008). http://dx.doi.org/10.5170/ CERN-2008-001.

A. M. Sirunyan, ${ }^{1}$ A. Tumasyan, ${ }^{1}$ W. Adam, ${ }^{2}$ F. Ambrogi, ${ }^{2}$ E. Asilar, ${ }^{2}$ T. Bergauer, ${ }^{2}$ J. Brandstetter, ${ }^{2}$ M. Dragicevic, ${ }^{2}$ J. Erö, ${ }^{2}$ A. Escalante Del Valle, ${ }^{2}$ M. Flechl, ${ }^{2}$ R. Frühwirth, ${ }^{2, b}$ V. M. Ghete, ${ }^{2}$ J. Hrubec, ${ }^{2}$ M. Jeitler, ${ }^{2, b}$ N. Krammer, ${ }^{2}$ I. Krätschmer, ${ }^{2}$ D. Liko, ${ }^{2}$ T. Madlener, ${ }^{2}$ I. Mikulec, ${ }^{2}$ N. Rad, ${ }^{2}$ H. Rohringer, ${ }^{2}$ J. Schieck, ${ }^{2, b}$ R. Schöfbeck, ${ }^{2}$ M. Spanring, ${ }^{2}$ D. Spitzbart, ${ }^{2}$ A. Taurok, ${ }^{2}$ W. Waltenberger, ${ }^{2}$ J. Wittmann, ${ }^{2}$ C.-E. Wulz, ${ }^{2, b}$ M. Zarucki, ${ }^{2}$ V. Chekhovsky, ${ }^{3}$ V. Mossolov, ${ }^{3}$ J. Suarez Gonzalez, ${ }^{3}$ E. A. De Wolf, ${ }^{4}$ D. Di Croce, ${ }^{4}$ X. Janssen, ${ }^{4}$ J. Lauwers, ${ }^{4}$ M. Pieters ${ }^{4}$ H. Van Haevermaet, ${ }^{4}$ P. Van Mechelen, ${ }^{4}$ N. Van Remortel, ${ }^{4}$ S. Abu Zeid, ${ }^{5}$ F. Blekman, ${ }^{5}$ J. D'Hondt, ${ }^{5}$ I. De Bruyn, ${ }^{5}$ J. De Clercq, ${ }^{5}$ K. Deroover, ${ }^{5}$ G. Flouris, ${ }^{5}$ D. Lontkovskyi, ${ }^{5}$ S. Lowette, ${ }^{5}$ I. Marchesini, ${ }^{5}$ S. Moortgat, ${ }^{5}$ L. Moreels, ${ }^{5}$ Q. Python,${ }^{5}$ K. Skovpen, ${ }^{5}$ S. Tavernier, ${ }^{5}$ W. Van Doninck, ${ }^{5}$ P. Van Mulders, ${ }^{5}$ I. Van Parijs, ${ }^{5}$ D. Beghin, ${ }^{6}$ B. Bilin, ${ }^{6}$ H. Brun, ${ }^{6}$ B. Clerbaux ${ }^{6}$ G. De Lentdecker, ${ }^{6}$ H. Delannoy, ${ }^{6}$ B. Dorney, ${ }^{6}$ G. Fasanella, ${ }^{6}$ L. Favart, ${ }^{6}$ R. Goldouzian, ${ }^{6}$ A. Grebenyuk, ${ }^{6}$ A. K. Kalsi, ${ }^{6}$ T. Lenzi, ${ }^{6}$ J. Luetic, ${ }^{6}$ N. Postiau, ${ }^{6}$ E. Starling, ${ }^{6}$ L. Thomas, ${ }^{6}$ C. Vander Velde, ${ }^{6}$ P. Vanlaer, ${ }^{6}$ D. Vannerom, ${ }^{6}$ Q. Wang, ${ }^{6}$ T. Cornelis, ${ }^{7}$ D. Dobur, ${ }^{7}$ A. Fagot, ${ }^{7}$ M. Gul, ${ }^{7}$ I. Khvastunov, ${ }^{7, c}$ D. Poyraz, ${ }^{7}$ C. Roskas, ${ }^{7}$ D. Trocino, ${ }^{7}$ M. Tytgat, ${ }^{7}$ W. Verbeke, ${ }^{7}$ B. Vermassen, ${ }^{7}$ M. Vit, ${ }^{7}$ N. Zaganidis, ${ }^{7}$ H. Bakhshiansohi, ${ }^{8}$ O. Bondu, ${ }^{8}$ S. Brochet, ${ }^{8}$ G. Bruno,${ }^{8}$ C. Caputo, ${ }^{8}$ P. David, ${ }^{8}$ C. Delaere,${ }^{8}$ M. Delcourt ${ }^{8}$ B. Francois, ${ }^{8}$ A. Giammanco, ${ }^{8}$ G. Krintiras, ${ }^{8}$ V. Lemaitre, ${ }^{8}$ A. Magitteri, ${ }^{8}$ A. Mertens, ${ }^{8}$ M. Musich, ${ }^{8}$ K. Piotrzkowski, ${ }^{8}$ A. Saggio, ${ }^{8}$ M. Vidal Marono, ${ }^{8}$ S. Wertz, ${ }^{8}$ J. Zobec, ${ }^{8}$ F. L. Alves, ${ }^{9}$ G. A. Alves,${ }^{9}$ M. Correa Martins Junior, ${ }^{9}$ G. Correia Silva, ${ }^{9}$ C. Hensel, ${ }^{9}$ A. Moraes, ${ }^{9}$ M. E. Pol, ${ }^{9}$ P. Rebello Teles, ${ }^{9}$

E. Belchior Batista Das Chagas, ${ }^{10}$ W. Carvalho, ${ }^{10}$ J. Chinellato, ${ }^{10, \mathrm{~d}}$ E. Coelho, ${ }^{10}$ E. M. Da Costa, ${ }^{10}$ G. G. Da Silveira, ${ }^{10, e}$ D. De Jesus Damiao, ${ }^{10}$ C. De Oliveira Martins,${ }^{10}$ S. Fonseca De Souza ${ }^{10}$ H. Malbouisson, ${ }^{10}$ D. Matos Figueiredo, ${ }^{10}$ M. Melo De Almeida, ${ }^{10}$ C. Mora Herrera, ${ }^{10}$ L. Mundim, ${ }^{10}$ H. Nogima, ${ }^{10}$ W. L. Prado Da Silva, ${ }^{10}$ L. J. Sanchez Rosas,${ }^{10}$ A. Santoro, ${ }^{10}$ A. Sznajder, ${ }^{10}$ M. Thiel,${ }^{10}$ E. J. Tonelli Manganote, ${ }^{10, d}$ F. Torres Da Silva De Araujo, ${ }^{10}$ A. Vilela Pereira, ${ }^{10}$ S. Ahuja ${ }^{11 \mathrm{a}}$ C. A. Bernardes, ${ }^{11 \mathrm{a}}$ L. Calligaris, ${ }^{11 \mathrm{a}}$ T. R. Fernandez Perez Tomei, ${ }^{11 \mathrm{a}}$ E. M. Gregores,${ }^{11 \mathrm{a}, 11 \mathrm{~b}}$ P. G. Mercadante, ${ }^{11 \mathrm{a}, 11 \mathrm{~b}}$ S. F. Novaes, ${ }^{11 \mathrm{a}}$ Sandra S. Padula, ${ }^{11 \mathrm{a}}$ A. Aleksandrov, ${ }^{12}$ R. Hadjiiska, ${ }^{12}$ P. Iaydjiev, ${ }^{12}$ A. Marinov, ${ }^{12}$ M. Misheva,${ }^{12}$ M. Rodozov, ${ }^{12}$ M. Shopova, ${ }^{12}$ G. Sultanov, ${ }^{12}$ A. Dimitrov, ${ }^{13}$ L. Litov, ${ }^{13}$ B. Pavlov, ${ }^{13}$ P. Petkov, ${ }^{13}$ W. Fang,,${ }^{14, f}$ X. Gao, ${ }^{14, \mathrm{f}}$ L. Yuan, ${ }^{14}$ M. Ahmad, ${ }^{15}$ J. G. Bian, ${ }^{15}$ G. M. Chen, ${ }^{15}$ H. S. Chen, ${ }^{15}$ M. Chen, ${ }^{15}$ Y. Chen, ${ }^{15}$ C. H. Jiang, ${ }^{15}$ D. Leggat ${ }^{15}$ H. Liao, ${ }^{15}$ Z. Liu, ${ }^{15}$ F. Romeo ${ }^{15}$ S. M. Shaheen, ${ }^{15, g}$ A. Spiezia, ${ }^{15}$ J. Tao, ${ }^{15}$ Z. Wang, ${ }^{15}$ E. Yazgan, ${ }^{15}$ H. Zhang,${ }^{15}$ S. Zhang, ${ }^{15, g}$ J. Zhao ${ }^{15}$ Y. Ban,${ }^{16}$ G. Chen, ${ }^{16}$ A. Levin, ${ }^{16} \mathrm{~J}$. Li, ${ }^{16}$ L. Li $,{ }^{16} \mathrm{Q} . \mathrm{Li},{ }^{16}$ Y. Mao, ${ }^{16} \mathrm{~S}$. J. Qian, ${ }^{16}$ D. Wang, ${ }^{16} \mathrm{Z} . \mathrm{Xu},{ }^{16}$ Y. Wang, ${ }^{17}$ C. Avila, ${ }^{18}$ A. Cabrera,${ }^{18}$ C. A. Carrillo Montoya, ${ }^{18}$ L. F. Chaparro Sierra, ${ }^{18}$ C. Florez, ${ }^{18}$

C. F. González Hernández, ${ }^{18}$ M. A. Segura Delgado, ${ }^{18}$ B. Courbon, ${ }^{19}$ N. Godinovic, ${ }^{19}$ D. Lelas, ${ }^{19}$ I. Puljak, ${ }^{19}$ T. Sculac, ${ }^{19}$ Z. Antunovic, ${ }^{20}$ M. Kovac, ${ }^{20}$ V. Brigljevic, ${ }^{21}$ D. Ferencek, ${ }^{21}$ K. Kadija, ${ }^{21}$ B. Mesic, ${ }^{21}$ A. Starodumov, ${ }^{21, h}$ T. Susa, ${ }^{21}$ M. W. Ather, ${ }^{22}$ A. Attikis, ${ }^{22}$ M. Kolosova, ${ }^{22}$ G. Mavromanolakis, ${ }^{22}$ J. Mousa, ${ }^{22}$ C. Nicolaou, ${ }^{22}$ F. Ptochos, ${ }^{22}$ P. A. Razis, ${ }^{22}$ H. Rykaczewski, ${ }^{22}$ M. Finger, ${ }^{23, i}$ M. Finger Jr., ${ }^{23, i}$ E. Ayala, ${ }^{24}$ E. Carrera Jarrin, ${ }^{25}$ H. Abdalla, ${ }^{26, j}$ A. A. Abdelalim, ${ }^{26, k, 1}$ E. Salama, ${ }^{26, m, n}$ S. Bhowmik,${ }^{27}$ A. Carvalho Antunes De Oliveira, ${ }^{27}$ R. K. Dewanjee, ${ }^{27}$ K. Ehataht, ${ }^{27}$ M. Kadastik, ${ }^{27}$ M. Raidal, ${ }^{27}$ C. Veelken, ${ }^{27}$ P. Eerola ${ }^{28}$ H. Kirschenmann, ${ }^{28}$ J. Pekkanen, ${ }^{28}$ M. Voutilainen, ${ }^{28}$ J. Havukainen, ${ }^{29}$ J. K. Heikkilä, ${ }^{29}$ T. Järvinen, ${ }^{29}$ V. Karimäki, ${ }^{29}$ R. Kinnunen, ${ }^{29}$ T. Lampén, ${ }^{29}$ K. Lassila-Perini, ${ }^{29}$ S. Laurila, ${ }^{29}$ S. Lehti, ${ }^{29}$ T. Lindén, ${ }^{29}$ P. Luukka, ${ }^{29}$ T. Mäenpää, ${ }^{29}$ H. Siikonen, ${ }^{29}$ E. Tuominen, ${ }^{29}$ J. Tuominiemi, ${ }^{29}$ T. Tuuva, ${ }^{30}$ M. Besancon, ${ }^{31}$ F. Couderc, ${ }^{31}$ M. Dejardin, ${ }^{31}$ D. Denegri, ${ }^{31}$ J. L. Faure, ${ }^{31}$ F. Ferri, ${ }^{31}$ S. Ganjour, ${ }^{31}$ A. Givernaud ${ }^{31}$ P. Gras, ${ }^{31}$ G. Hamel de Monchenault, ${ }^{31}$ P. Jarry, ${ }^{31}$ C. Leloup ${ }^{31}$ E. Locci ${ }^{31}$ J. Malcles,${ }^{31}$ G. Negro, ${ }^{31}$ J. Rander, ${ }^{31}$ A. Rosowsky, ${ }^{31}$ M. Ö. Sahin, ${ }^{31}$ M. Titov, ${ }^{31}$ A. Abdulsalam, ${ }^{32,0}$ C. Amendola, ${ }^{32}$ I. Antropov, ${ }^{32}$ F. Beaudette, ${ }^{32}$ P. Busson,${ }^{32}$ C. Charlot, ${ }^{32}$ 
R. Granier de Cassagnac, ${ }^{32}$ I. Kucher, ${ }^{32}$ A. Lobanov, ${ }^{32}$ J. Martin Blanco, ${ }^{32}$ C. Martin Perez, ${ }^{32}$ M. Nguyen, ${ }^{32}$ C. Ochando, ${ }^{32}$ G. Ortona, ${ }^{32}$ P. Paganini, ${ }^{32}$ P. Pigard, ${ }^{32}$ J. Rembser, ${ }^{32}$ R. Salerno, ${ }^{32}$ J. B. Sauvan, ${ }^{32}$ Y. Sirois, ${ }^{32}$ A. G. Stahl Leiton, ${ }^{32}$ A. Zabi, ${ }^{32}$ A. Zghiche, ${ }^{32}$ J.-L. Agram, ${ }^{33, p}$ J. Andrea, ${ }^{33}$ D. Bloch, ${ }^{33}$ J.-M. Brom, ${ }^{33}$ E. C. Chabert, ${ }^{33}$ V. Cherepanov, ${ }^{33}$ C. Collard, ${ }^{33}$ E. Conte, ${ }^{33, p}$ J.-C. Fontaine, ${ }^{33, p}$ D. Gelé, ${ }^{33}$ U. Goerlach, ${ }^{33}$ M. Jansová, ${ }^{33}$ A.-C. Le Bihan, ${ }^{33}$ N. Tonon, ${ }^{33}$ P. Van Hove, ${ }^{33}$ S. Gadrat, ${ }^{34}$ S. Beauceron, ${ }^{35}$ C. Bernet, ${ }^{35}$ G. Boudoul,${ }^{35}$ N. Chanon, ${ }^{35}$ R. Chierici, ${ }^{35}$ D. Contardo, ${ }^{35}$ P. Depasse, ${ }^{35}$ H. El Mamouni, ${ }^{35}$ J. Fay, ${ }^{35}$ L. Finco, ${ }^{35}$ S. Gascon, ${ }^{35}$ M. Gouzevitch, ${ }^{35}$ G. Grenier,${ }^{35}$ B. Ille, ${ }^{35}$ F. Lagarde,${ }^{35}$ I. B. Laktineh, ${ }^{35}$ H. Lattaud, ${ }^{35}$ M. Lethuillier, ${ }^{35}$ L. Mirabito, ${ }^{35}$ S. Perries, ${ }^{35}$ A. Popov, ${ }^{35, q}$ V. Sordini,,${ }^{35}$ G. Touquet, ${ }^{35}$ M. Vander Donckt, ${ }^{35}$ S. Viret, ${ }^{35}$ A. Khvedelidze, ${ }^{36, i}$ Z. Tsamalaidze, ${ }^{37, i}$ C. Autermann, ${ }^{38}$ L. Feld, ${ }^{38}$ M. K. Kiesel, ${ }^{38}$ K. Klein, ${ }^{38}$ M. Lipinski, ${ }^{38}$ M. Preuten, ${ }^{38}$ M. P. Rauch, ${ }^{38}$ C. Schomakers, ${ }^{38}$ J. Schulz, ${ }^{38}$ M. Teroerde, ${ }^{38}$ B. Wittmer, ${ }^{38}$ V. Zhukov, ${ }^{38, q}$ A. Albert, ${ }^{39}$ D. Duchardt, ${ }^{39}$ M. Endres, ${ }^{39}$ M. Erdmann,${ }^{39}$ S. Erdweg, ${ }^{39}$ T. Esch, ${ }^{39}$ R. Fischer, ${ }^{39}$ S. Ghosh,${ }^{39}$ A. Güth, ${ }^{39}$ T. Hebbeker, ${ }^{39}$ C. Heidemann, ${ }^{39}$ K. Hoepfner, ${ }^{39}$ H. Keller, ${ }^{39}$ L. Mastrolorenzo, ${ }^{39}$ M. Merschmeyer, ${ }^{39}$ A. Meyer, ${ }^{39}$ P. Millet, ${ }^{39}$ S. Mukherjee, ${ }^{39}$ T. Pook,${ }^{39}$ M. Radziej, ${ }^{39}$ H. Reithler, ${ }^{39}$ M. Rieger, ${ }^{39}$ A. Schmidt, ${ }^{39}$ D. Teyssier,${ }^{39}$ S. Thüer, ${ }^{39}$ G. Flügge, ${ }^{40}$ O. Hlushchenko, ${ }^{40}$ T. Kress, ${ }^{40}$ A. Künsken, ${ }^{40}$ T. Müller, ${ }^{40}$ A. Nehrkorn, ${ }^{40}$ A. Nowack,${ }^{40}$ C. Pistone, ${ }^{40}$ O. Pooth,${ }^{40}$ D. Roy ${ }^{40}$ H. Sert, ${ }^{40}$ A. Stahl, ${ }^{40, \mathrm{r}}$ M. Aldaya Martin, ${ }^{41}$ T. Arndt,${ }^{41}$ C. Asawatangtrakuldee, ${ }^{41}$ I. Babounikau, ${ }^{41}$ K. Beernaert ${ }^{41}$ O. Behnke, ${ }^{41}$ U. Behrens, ${ }^{41}$ A. Bermúdez Martínez ${ }^{41}$ D. Bertsche, ${ }^{41}$ A. A. Bin Anuar, ${ }^{41}$ K. Borras, ${ }^{41, s}$

V. Botta, ${ }^{41}$ A. Campbell, ${ }^{41}$ P. Connor, ${ }^{41}$ C. Contreras-Campana, ${ }^{41}$ V. Danilov, ${ }^{41}$ A. De Wit, ${ }^{41}$ M. M. Defranchis, ${ }^{41}$ C. Diez Pardos, ${ }^{41}$ D. Domínguez Damiani, ${ }^{41}$ G. Eckerlin, ${ }^{41}$ T. Eichhorn, ${ }^{41}$ A. Elwood, ${ }^{41}$ E. Eren, ${ }^{41}$ E. Gallo, ${ }^{41, t}$ A. Geiser, ${ }^{41}$

A. Grohsjean, ${ }^{41}$ M. Guthoff, ${ }^{41}$ M. Haranko, ${ }^{41}$ A. Harb,${ }^{41}$ J. Hauk, ${ }^{41}$ H. Jung, ${ }^{41}$ M. Kasemann, ${ }^{41}$ J. Keaveney, ${ }^{41}$ C. Kleinwort, ${ }^{41}$ J. Knolle, ${ }^{41}$ D. Krücker, ${ }^{41}$ W. Lange ${ }^{41}$ A. Lelek,${ }^{41}$ T. Lenz,${ }^{41}$ J. Leonard, ${ }^{41}$ K. Lipka, ${ }^{41}$ W. Lohmann, ${ }^{41, u}$ R. Mankel, ${ }^{41}$ I.-A. Melzer-Pellmann, ${ }^{41}$ A. B. Meyer, ${ }^{41}$ M. Meyer ${ }^{41}$ M. Missiroli, ${ }^{41}$ G. Mittag,${ }^{41}$ J. Mnich, ${ }^{41}$ V. Myronenko, ${ }^{41}$

S. K. Pflitsch ${ }^{41}$ D. Pitzl,${ }^{41}$ A. Raspereza,${ }^{41}$ M. Savitskyi, ${ }^{41}$ P. Saxena, ${ }^{41}$ P. Schütze, ${ }^{41}$ C. Schwanenberger, ${ }^{41}$

R. Shevchenko, ${ }^{41}$ A. Singh ${ }^{41}$ H. Tholen, ${ }^{41}$ O. Turkot, ${ }^{41}$ A. Vagnerini, ${ }^{41}$ G. P. Van Onsem, ${ }^{41}$ R. Walsh,${ }^{41}$ Y. Wen, ${ }^{41}$ K. Wichmann, ${ }^{41}$ C. Wissing, ${ }^{41}$ O. Zenaiev, ${ }^{41}$ R. Aggleton, ${ }^{42}$ S. Bein, ${ }^{42}$ L. Benato, ${ }^{42}$ A. Benecke,${ }^{42}$ V. Blobel, ${ }^{42}$ T. Dreyer,${ }^{42}$ E. Garutti, ${ }^{42}$ D. Gonzalez, ${ }^{42}$ P. Gunnellini, ${ }^{42}$ J. Haller, ${ }^{42}$ A. Hinzmann, ${ }^{42}$ A. Karavdina, ${ }^{42}$ G. Kasieczka, ${ }^{42}$ R. Klanner, ${ }^{42}$ R. Kogler ${ }^{42}$ N. Kovalchuk, ${ }^{42}$ S. Kurz, ${ }^{42}$ V. Kutzner, ${ }^{42}$ J. Lange, ${ }^{42}$ D. Marconi, ${ }^{42}$ J. Multhaup,${ }^{42}$ M. Niedziela, ${ }^{42}$ C. E. N. Niemeyer, ${ }^{42}$ D. Nowatschin, ${ }^{42}$ A. Perieanu, ${ }^{42}$ A. Reimers, ${ }^{42}$ O. Rieger, ${ }^{42}$ C. Scharf, ${ }^{42}$ P. Schleper, ${ }^{42}$ S. Schumann, ${ }^{42}$ J. Schwandt, ${ }^{42}$ J. Sonneveld, ${ }^{42}$ H. Stadie, ${ }^{42}$ G. Steinbrück, ${ }^{42}$ F. M. Stober, ${ }^{42}$ M. Stöver, ${ }^{42}$ A. Vanhoefer ${ }^{42}$ B. Vormwald, ${ }^{42}$ I. Zoi, ${ }^{42}$ M. Akbiyik, ${ }^{43}$ C. Barth, ${ }^{43}$ M. Baselga, ${ }^{43}$ S. Baur, ${ }^{43}$ E. Butz, ${ }^{43}$ R. Caspart, ${ }^{43}$ T. Chwalek, ${ }^{43}$ F. Colombo, ${ }^{43}$ W. De Boer, ${ }^{43}$ A. Dierlamm, ${ }^{43}$ K. El Morabit ${ }^{43}$ N. Faltermann, ${ }^{43}$ B. Freund, ${ }^{43}$ M. Giffels, ${ }^{43}$ M. A. Harrendorf, ${ }^{43}$ F. Hartmann, ${ }^{43, \mathrm{r}}$ S. M. Heindl, ${ }^{43}$ U. Husemann, ${ }^{43}$ F. Kassel, ${ }^{43, \mathrm{r}}$ I. Katkov, ${ }^{43, \mathrm{q}}$ S. Kudella, ${ }^{43}$ H. Mildner, ${ }^{43}$ S. Mitra, ${ }^{43}$ M. U. Mozer, ${ }^{43}$ Th. Müller, ${ }^{43}$ M. Plagge, ${ }^{43}$ G. Quast, ${ }^{43}$ K. Rabbertz, ${ }^{43}$ M. Schröder, ${ }^{43}$ I. Shvetsov, ${ }^{43}$ G. Sieber, ${ }^{43}$ H. J. Simonis, ${ }^{43}$ R. Ulrich, ${ }^{43}$ S. Wayand, ${ }^{43}$ M. Weber, ${ }^{43}$ T. Weiler, ${ }^{43}$ S. Williamson, ${ }^{43}$ C. Wöhrmann, ${ }^{43}$ R. Wolf, ${ }^{43}$ G. Anagnostou, ${ }^{44}$ G. Daskalakis, ${ }^{44}$ T. Geralis, ${ }^{44}$ A. Kyriakis, ${ }^{44}$ D. Loukas, ${ }^{44}$ G. Paspalaki, ${ }^{44}$ I. Topsis-Giotis, ${ }^{44}$ G. Karathanasis, ${ }^{45}$ S. Kesisoglou, ${ }^{45}$ P. Kontaxakis, ${ }^{45}$ A. Panagiotou, ${ }^{45}$ I. Papavergou, ${ }^{45}$ N. Saoulidou, ${ }^{45}$ E. Tziaferi ${ }^{45}$ K. Vellidis ${ }^{45}$ K. Kousouris, ${ }^{46}$ I. Papakrivopoulos,${ }^{46}$ G. Tsipolitis, ${ }^{46}$ I. Evangelou, ${ }^{47}$ C. Foudas, ${ }^{47}$ P. Gianneios,${ }^{47}$ P. Katsoulis, ${ }^{47}$ P. Kokkas, ${ }^{47}$ S. Mallios ${ }^{47}$ N. Manthos, ${ }^{47}$ I. Papadopoulos, ${ }^{47}$ E. Paradas, ${ }^{47}$ J. Strologas, ${ }^{47}$ F. A. Triantis,${ }^{47}$ D. Tsitsonis, ${ }^{47}$ M. Bartók, ${ }^{48, v}$ M. Csanad ${ }^{48}$ N. Filipovic, ${ }^{48}$ P. Major,${ }^{48}$ M. I. Nagy, ${ }^{48}$ G. Pasztor, ${ }^{48}$ O. Surányi, ${ }^{48}$ G. I. Veres ${ }^{48}$ G. Bencze ${ }^{49}$ C. Hajdu, ${ }^{49}$ D. Horvath, ${ }^{49, w}$ Á. Hunyadi, ${ }^{49}$ F. Sikler ${ }^{49}$ T. Á. Vámi ${ }^{49}$ V. Veszpremi, ${ }^{49}$ G. Vesztergombi, ${ }^{49, a, v}$ N. Beni, ${ }^{50}$ S. Czellar, ${ }^{50}$ J. Karancsi,${ }^{50, x}$ A. Makovec,${ }^{50}$ J. Molnar, ${ }^{50}$ Z. Szillasi, ${ }^{50}$ P. Raics,${ }^{51}$ Z. L. Trocsanyi, ${ }^{51}$ B. Ujvari, ${ }^{51}$ S. Choudhury, ${ }^{52}$ J. R. Komaragiri, ${ }^{52}$ P. C. Tiwari, ${ }^{52}$ S. Bahinipati, ${ }^{53, y}$ C. Kar, ${ }^{53}$ P. Mal,${ }^{53}$ K. Mandal, ${ }^{53}$ A. Nayak, ${ }^{53, z}$ D. K. Sahoo, ${ }^{53, y}$ S. K. Swain, ${ }^{53}$ S. Bansal, ${ }^{54}$ S. B. Beri,${ }^{54}$ V. Bhatnagar, ${ }^{54}$ S. Chauhan, ${ }^{54}$ R. Chawla, ${ }^{54}$ N. Dhingra, ${ }^{54}$ R. Gupta, ${ }^{54}$ A. Kaur, ${ }^{54}$ M. Kaur, ${ }^{54}$ S. Kaur, ${ }^{54}$ R. Kumar, ${ }^{54}$ P. Kumari,${ }^{54}$ M. Lohan, ${ }^{54}$ A. Mehta, ${ }^{54}$ K. Sandeep,${ }^{54}$ S. Sharma,${ }^{54}$ J. B. Singh, ${ }^{54}$ A. K. Virdi, ${ }^{54}$ G. Walia ${ }^{54}$ A. Bhardwaj, ${ }^{55}$ B. C. Choudhary,${ }^{55}$ R. B. Garg, ${ }^{55}$ M. Gola, ${ }^{55}$ S. Keshri, ${ }^{55}$ Ashok Kumar, ${ }^{55}$ S. Malhotra, ${ }^{55}$ M. Naimuddin, ${ }^{55}$ P. Priyanka ${ }^{55}$ K. Ranjan, ${ }^{55}$ Aashaq Shah ${ }^{55}$ R. Sharma, ${ }^{55}$ R. Bhardwaj, ${ }^{56, a a}$ M. Bharti, ${ }^{56, a a}$ R. Bhattacharya, ${ }^{56}$ S. Bhattacharya,${ }^{56}$ U. Bhawandeep, ${ }^{56, \text { aa }}$ D. Bhowmik, ${ }^{56}$ S. Dey, ${ }^{56}$ S. Dutt,${ }^{56, a a}$ S. Dutta ${ }^{56}$ S. Ghosh, ${ }^{56}$ K. Mondal, ${ }^{56}$ S. Nandan, ${ }^{56}$ A. Purohit, ${ }^{56}$ P. K. Rout,${ }^{56}$ A. Roy, ${ }^{56}$ S. Roy Chowdhury, ${ }^{56}$ G. Saha, ${ }^{56}$ S. Sarkar, ${ }^{56}$ M. Sharan, ${ }^{56}$ B. Singh, ${ }^{56, \text { aa }}$ S. Thakur, ${ }^{56, \text { aa }}$ P. K. Behera,${ }^{57}$ R. Chudasama, ${ }^{58}$ D. Dutta, ${ }^{58}$ V. Jha, ${ }^{58}$ V. Kumar, ${ }^{58}$

P. K. Netrakanti, ${ }^{58}$ L. M. Pant, ${ }^{58}$ P. Shukla, ${ }^{58}$ T. Aziz,${ }^{59}$ M. A. Bhat, ${ }^{59}$ S. Dugad,${ }^{59}$ G. B. Mohanty, ${ }^{59}$ N. Sur, ${ }^{59}$ B. Sutar, ${ }^{59}$ Ravindra Kumar Verma, ${ }^{59}$ S. Banerjee, ${ }^{60}$ S. Bhattacharya, ${ }^{60}$ S. Chatterjee,${ }^{60}$ P. Das, ${ }^{60}$ M. Guchait, ${ }^{60}$ Sa. Jain, ${ }^{60}$ 
S. Karmakar, ${ }^{60}$ S. Kumar, ${ }^{60}$ M. Maity, ${ }^{60, b b}$ G. Majumder, ${ }^{60}$ K. Mazumdar, ${ }^{60}$ N. Sahoo, ${ }^{60}$ T. Sarkar, ${ }^{60, b b}$ S. Chauhan, ${ }^{61}$ S. Dube, ${ }^{61}$ V. Hegde,${ }^{61}$ A. Kapoor,${ }^{61}$ K. Kothekar, ${ }^{61}$ S. Pandey, ${ }^{61}$ A. Rane,${ }^{61}$ S. Sharma, ${ }^{61}$ S. Chenarani, ${ }^{62, c c}$ E. Eskandari Tadavani, ${ }^{62}$ S. M. Etesami, ${ }^{62, c c}$ M. Khakzad, ${ }^{62}$ M. Mohammadi Najafabadi, ${ }^{62}$ M. Naseri, ${ }^{62}$

F. Rezaei Hosseinabadi, ${ }^{62}$ B. Safarzadeh, ${ }^{62 \text { dd }}$ M. Zeinali, ${ }^{62}$ M. Felcini, ${ }^{63}$ M. Grunewald, ${ }^{63}$ M. Abbrescia, ${ }^{64 a, 64 b}$ C. Calabria, ${ }^{64 a, 64 b}$ A. Colaleo, ${ }^{64 a}$ D. Creanza, ${ }^{64 a, 64 c}$ L. Cristella,${ }^{64 a, 64 b}$ N. De Filippis, ${ }^{64 a, 64 c}$ M. De Palma, ${ }^{64 a, 64 b}$ A. Di Florio ${ }^{64 a, 64 b}$ F. Errico, ${ }^{64 a, 64 b}$ L. Fiore ${ }^{64 a}$ A. Gelmi ${ }^{64 a, 64 b}$ G. Iaselli, ${ }^{64 a, 64 c}$ M. Ince, ${ }^{64 a, 64 b}$ S. Lezki, ${ }^{64 a, 64 b}$ G. Maggi ${ }^{64 a, 64 c}$ M. Maggi, ${ }^{64 a}$ G. Miniello, ${ }^{64 a, 64 b}$ S. My, ${ }^{64 a, 64 b}$ S. Nuzzo, ${ }^{64 a, 64 b}$ A. Pompili, ${ }^{64 a, 64 b}$ G. Pugliese,${ }^{64 a, 64 c}$ R. Radogna, ${ }^{64 a}$ A. Ranieri, ${ }^{64 a}$ G. Selvaggi, ${ }^{64 a 64 b}$ A. Sharma ${ }^{64 a}$ L. Silvestris, ${ }^{64 a}$ R. Venditti, ${ }^{64 a}$ P. Verwilligen, ${ }^{64 a}$ G. Zito, ${ }^{64 a}$ G. Abbiendi, ${ }^{65 a}$ C. Battilana ${ }^{65 a, 65 b}$ D. Bonacorsi, ${ }^{65 a, 65 b}$ L. Borgonovi ${ }^{65 a, 65 b}$ S. Braibant-Giacomelli, ${ }^{65 a, 65 b}$ R. Campanini, ${ }^{65 a, 65 b}$ P. Capiluppi ${ }^{65 a, 65 b}$ A. Castro, ${ }^{65 a, 65 b}$ F. R. Cavallo, ${ }^{65 a}$ S. S. Chhibra,${ }^{65 a, 65 b}$ C. Ciocca, ${ }^{65 a}$ G. Codispoti, ${ }^{65 a, 65 b}$ M. Cuffiani, ${ }^{65 a, 65 b}$ G. M. Dallavalle, ${ }^{65 a}$ F. Fabbri, ${ }^{65 a}$ A. Fanfani, ${ }^{65 a, 65 b}$ E. Fontanesi ${ }^{65 a}$ P. Giacomelli, ${ }^{65 a}$ C. Grandi, ${ }^{65 a}$ L. Guiducci, ${ }^{65 a, 65 b}$

F. Iemmi,${ }^{65 a 65 b}$ S. Lo Meo, ${ }^{65 a}$ S. Marcellini, ${ }^{65 a}$ G. Masetti ${ }^{65 a}$ A. Montanari, ${ }^{65 a}$ F. L. Navarria, ${ }^{65 a, 65 b}$ A. Perrotta ${ }^{65 a}$ F. Primavera, ${ }^{65 a, 65 b, r}$ T. Rovelli, ${ }^{65 a, 65 b}$ G. P. Siroli, ${ }^{65 a, 65 b}$ N. Tosi, ${ }^{65 a}$ S. Albergo, ${ }^{66 a, 66 b}$ A. Di Mattia, ${ }^{66 a}$ R. Potenza, ${ }^{66 a, 66 b}$ A. Tricomi ${ }^{66 a, 66 b}$ C. Tuve, ${ }^{66 a, 66 b}$ G. Barbagli, ${ }^{67 a}$ K. Chatterjee, ${ }^{67,67 b}$ V. Ciulli, ${ }^{67,67 b}$ C. Civinini, ${ }^{67 a}$ R. D'Alessandro, ${ }^{67 a, 67 b}$ E. Focardi, ${ }^{67 a, 67 b}$ G. Latino, ${ }^{67 a}$ P. Lenzi, ${ }^{67 a, 67 b}$ M. Meschini, ${ }^{67 a}$ S. Paoletti, ${ }^{67 a}$ L. Russo, ${ }^{67 a, e e}$ G. Sguazzoni, ${ }^{67 a}$ D. Strom, ${ }^{67 a}$ L. Viliani, ${ }^{67 a}$ L. Benussi ${ }^{68}$ S. Bianco,${ }^{68}$ F. Fabbri, ${ }^{68}$ D. Piccolo, ${ }^{68}$ F. Ferro ${ }^{69 a}$ F. Ravera, ${ }^{69,69 b}$ E. Robutti, ${ }^{69 a}$ S. Tosi, ${ }^{69 a, 69 b}$ A. Benaglia, ${ }^{70 \mathrm{a}}$ A. Beschi, ${ }^{70 a, 70 \mathrm{~b}}$ L. Brianza, ${ }^{70 a, 70 \mathrm{~b}}$ F. Brivio, ${ }^{70 a, 70 \mathrm{~b}}$ V. Ciriolo,${ }^{70 a, 70 b, \mathrm{r}}$ S. Di Guida,${ }^{70 \mathrm{a}, 70 \mathrm{~b}, \mathrm{r}}$ M. E. Dinardo, ${ }^{70 a, 70 \mathrm{~b}}$ S. Fiorendi, ${ }^{70 a, 70 b}$ S. Gennai, ${ }^{70 a}$ A. Ghezzi, ${ }^{70 a, 70 b}$ P. Govoni, ${ }^{70 a, 70 b}$ M. Malberti, ${ }^{70 a, 70 b}$ S. Malvezzi, ${ }^{70 a}$ A. Massironi, ${ }^{70 a, 70 b}$ D. Menasce ${ }^{70 a}$ F. Monti, ${ }^{70 a}$ L. Moroni, ${ }^{70 a}$ M. Paganoni, ${ }^{70 a, 70 b}$ D. Pedrini, ${ }^{70 a}$ S. Ragazzi, ${ }^{70 a, 70 b}$ T. Tabarelli de Fatis, ${ }^{70 a, 70 b}$ D. Zuolo, ${ }^{70 a, 70 b}$ S. Buontempo, ${ }^{71 a}$ N. Cavallo, ${ }^{71 a, 71 c}$ A. Di Crescenzo,${ }^{71 a, 71 b}$ F. Fabozzi, ${ }^{71 a, 71 c}$ F. Fienga, ${ }^{71 a}$ G. Galati, ${ }^{71 a}$ A. O. M. Iorio, ${ }^{71 a, 71 b}$ W. A. Khan, ${ }^{71 a}$ L. Lista, ${ }^{71 a}$ S. Meola, ${ }^{71 a, 71 d, r}$ P. Paolucci, ${ }^{71 a, r}$ C. Sciacca, ${ }^{71 a, 71 b}$ E. Voevodina, ${ }^{71 a, 71 b}$ P. Azzi, ${ }^{72 a}$ N. Bacchetta, ${ }^{72 a}$ D. Bisello, ${ }^{72 a, 72 b}$ A. Boletti, ${ }^{72 a, 72 b}$ A. Bragagnolo, ${ }^{72 a}$ R. Carlin, ${ }^{72 a, 72 b}$ P. Checchia, ${ }^{72 a}$ M. Dall'Osso, ${ }^{72 a, 72 b}$ P. De Castro Manzano, ${ }^{72 a}$ T. Dorigo, ${ }^{72 a}$ U. Dosselli, ${ }^{72 a}$ F. Gasparini, ${ }^{72 a, 72 b}$ U. Gasparini, ${ }^{72 a, 72 b}$ A. Gozzelino, ${ }^{72 a}$ S. Y. Hoh, ${ }^{72 a}$ S. Lacaprara, ${ }^{72 a}$ P. Lujan, ${ }^{72 a}$ M. Margoni, ${ }^{72 a, 72 b}$ A. T. Meneguzzo, ${ }^{72 a, 72 b}$ J. Pazzini, ${ }^{72 a, 72 b}$ P. Ronchese, ${ }^{72 a, 72 b}$ R. Rossin, ${ }^{72 a, 72 b}$ F. Simonetto, ${ }^{72 a, 72 b}$ A. Tiko, ${ }^{72 a}$ E. Torassa, ${ }^{72 a}$ M. Zanetti, ${ }^{72 a, 72 b}$ P. Zotto, ${ }^{72 a, 72 b}$ G. Zumerle, ${ }^{72 a, 72 b}$ A. Braghieri, ${ }^{73 a}$ A. Magnani, ${ }^{73 a}$ P. Montagna, ${ }^{73 a, 73 b}$ S. P. Ratti, ${ }^{73 a, 73 b}$ V. Re, ${ }^{73 a}$ M. Ressegotti,${ }^{73 a, 73 b}$ C. Riccardi, ${ }^{73 a, 73 b}$ P. Salvini, ${ }^{73 a}$ I. Vai, ${ }^{73 a, 73 b}$ P. Vitulo, ${ }^{73 a, 73 b}$ M. Biasini, ${ }^{74 a, 74 b}$ G. M. Bilei, ${ }^{74 a}$ C. Cecchi, ${ }^{74 a, 74 b}$ D. Ciangottini, ${ }^{74 a, 74 b}$ L. Fanò, ${ }^{74 a, 74 b}$ P. Lariccia, ${ }^{74 a, 74 b}$ R. Leonardi, ${ }^{74 a, 74 b}$ E. Manoni, ${ }^{74 a}$ G. Mantovani, ${ }^{74 a, 74 b}$ V. Mariani, ${ }^{74 a, 74 b}$ M. Menichelli, ${ }^{74 a}$ A. Rossi, ${ }^{74 a}, 74 b$ A. Santocchia $,{ }^{74 a}, 74 b$ D. Spiga, ${ }^{74 a}$ K. Androsov,${ }^{75 a}$ P. Azzurri, ${ }^{75 a}$ G. Bagliesi, ${ }^{75 a}$ L. Bianchini, ${ }^{75 a}$ T. Boccali, ${ }^{75 a}$ L. Borrello, ${ }^{75 a}$ R. Castaldi, ${ }^{75 a}$ M. A. Ciocci, ${ }^{75 a, 75 b}$ R. Dell'Orso, ${ }^{75 a}$ G. Fedi, ${ }^{75 a}$ F. Fiori, ${ }^{75 a, 75 c}$ L. Giannini, ${ }^{75 a, 75 c}$ A. Giassi, ${ }^{75 a}$ M. T. Grippo, ${ }^{75 a}$ F. Ligabue, ${ }^{75 a, 75 c}$ E. Manca,${ }^{75 a, 75 c}$ G. Mandorli, ${ }^{75 a, 75 c}$ A. Messineo, ${ }^{75 a, 75 b}$ F. Palla, ${ }^{75 a}$ A. Rizzi, ${ }^{75 a, 75 b}$ P. Spagnolo, ${ }^{75 a}$ R. Tenchini, ${ }^{75 a}$ G. Tonelli, ${ }^{75 a, 75 b}$ A. Venturi, ${ }^{75 a}$ P. G. Verdini, ${ }^{75 a}$ L. Barone, ${ }^{76 a, 76 b}$ F. Cavallari, ${ }^{76 \mathrm{a}}$ M. Cipriani, ${ }^{76 a, 76 b}$ D. Del Re, ${ }^{76 a, 76 b}$ E. Di Marco,${ }^{76 a, 76 b}$ M. Diemoz ${ }^{76 a}$ S. Gelli, ${ }^{76 a, 76 b}$ E. Longo, ${ }^{76 a, 76 b}$ B. Marzocchi, ${ }^{76 a, 76 b}$ P. Meridiani, ${ }^{76 a}$ G. Organtini, ${ }^{76 a, 76 b}$ F. Pandolfi, ${ }^{76 a}$ R. Paramatti,${ }^{76 a, 76 b}$ F. Preiato, ${ }^{76 a, 76 b}$ S. Rahatlou, ${ }^{76 a, 76 b}$ C. Rovelli, ${ }^{76 \mathrm{a}}$ F. Santanastasio, ${ }^{76 \mathrm{a}, 76 \mathrm{~b}}$ N. Amapane,${ }^{77,77 \mathrm{~b}}$ R. Arcidiacono, ${ }^{77 \mathrm{a}, 77 \mathrm{c}}$ S. Argiro, ${ }^{77,77 \mathrm{~b}}$ M. Arneodo, ${ }^{77 \mathrm{a}, 77 \mathrm{c}}$ N. Bartosik, ${ }^{77 a}$ R. Bellan, ${ }^{77,77 b}$ C. Biino, ${ }^{77 a}$ N. Cartiglia, ${ }^{77 a}$ F. Cenna, ${ }^{77 a, 77 b}$ S. Cometti, ${ }^{77 a}$ M. Costa, ${ }^{77 a, 77 b}$ R. Covarelli, ${ }^{77 a, 77 b}$ N. Demaria, ${ }^{77 a}$ B. Kiani, ${ }^{77 a, 77 b}$ C. Mariotti, ${ }^{77 a}$ S. Maselli, ${ }^{77 a}$ E. Migliore, ${ }^{77 a, 77 b}$ V. Monaco, ${ }^{77 a, 77 b}$ E. Monteil, ${ }^{77,77 b}$ M. Monteno, ${ }^{77 a}$ M. M. Obertino, ${ }^{77,77 b}$ L. Pacher, ${ }^{77,77 b}$ N. Pastrone ${ }^{77 a}$ M. Pelliccioni, ${ }^{77 a}$ G. L. Pinna Angioni, ${ }^{77,77 b}$ A. Romero, ${ }^{77 a, 77 b}$ M. Ruspa,${ }^{77 a, 77 c}$ R. Sacchi, ${ }^{77 a, 77 b}$ K. Shchelina, ${ }^{77 a, 77 b}$ V. Sola, ${ }^{77 a}$ A. Solano, ${ }^{77,77 b}$ D. Soldi, ${ }^{77,77 b}$ A. Staiano, ${ }^{77 a}$ S. Belforte, ${ }^{78 a}$ V. Candelise,${ }^{78 a, 78 b}$ M. Casarsa, ${ }^{78 a}$ F. Cossutti, ${ }^{78 a}$ A. Da Rold ${ }^{78 a, 78 b}$ G. Della Ricca ${ }^{78 a, 78 b}$ F. Vazzoler, ${ }^{78 a, 78 b}$ A. Zanetti, ${ }^{78 a}$ D. H. Kim, ${ }^{79}$ G. N. Kim, ${ }^{79}$ M. S. Kim, ${ }^{79}$ J. Lee, ${ }^{79}$ S. Lee, ${ }^{79}$ S. W. Lee, ${ }^{79}$ C. S. Moon, ${ }^{79}$ Y. D. Oh, ${ }^{79}$ S. I. Pak, ${ }^{79}$ S. Sekmen, ${ }^{79}$ D. C. Son,${ }^{79}$ Y. C. Yang, ${ }^{79}$ H. Kim, ${ }^{80}$ D. H. Moon, ${ }^{80}$ G. Oh, ${ }^{80}$ J. Goh, ${ }^{81, f f}$ T. J. Kim, ${ }^{81}$ S. Cho, ${ }^{82}$ S. Choi, ${ }^{82}$ Y. Go, ${ }^{82}$ D. Gyun, ${ }^{82}$ S. Ha, ${ }^{82}$ B. Hong, ${ }^{82}$ Y. Jo ${ }^{82}$ K. Lee,${ }^{82}$ K. S. Lee, ${ }^{82}$ S. Lee, ${ }^{82}$ J. Lim, ${ }^{82}$ S. K. Park, ${ }^{82}$ Y. Roh, ${ }^{82}$ H. S. Kim, ${ }^{83}$ J. Almond, ${ }^{84}$ J. Kim, ${ }^{84}$ J. S. Kim, ${ }^{84}$ H. Lee, ${ }^{84}$ K. Lee,${ }^{84}$ K. Nam, ${ }^{84}$ S. B. Oh, ${ }^{84}$ B. C. Radburn-Smith, ${ }^{84}$ S. h. Seo,${ }^{84}$ U. K. Yang,${ }^{84}$ H. D. Yoo,${ }^{84}$ G. B. Yu ${ }^{84}$ D. Jeon, ${ }^{85}$ H. Kim ${ }^{85}$ J. H. Kim, ${ }^{85}$ J. S. H. Lee, ${ }^{85}$ I. C. Park,${ }^{85}$ Y. Choi, ${ }^{86}$ C. Hwang, ${ }^{86}$ J. Lee, ${ }^{86}$ I. Yu, ${ }^{86}$ V. Dudenas, ${ }^{87}$ A. Juodagalvis, ${ }^{87}$ J. Vaitkus, ${ }^{87}$ I. Ahmed, ${ }^{88}$ Z. A. Ibrahim, ${ }^{88}$ M. A. B. Md Ali, ${ }^{88, g g}$ F. Mohamad Idris, ${ }^{88, \text { hh }}$ W. A. T. Wan Abdullah, ${ }^{88}$ M. N. Yusli, ${ }^{88}$ Z. Zolkapli, ${ }^{88}$ J. F. Benitez, ${ }^{89}$ A. Castaneda Hernandez, ${ }^{89}$ J. A. Murillo Quijada, ${ }^{89}$ H. Castilla-Valdez,${ }^{90}$ E. De La Cruz-Burelo, ${ }^{90}$ M. C. Duran-Osuna, ${ }^{90}$ I. Heredia-De La Cruz, ${ }^{90, i i}$ R. Lopez-Fernandez, ${ }^{90}$ J. Mejia Guisao, ${ }^{90}$ R. I. Rabadan-Trejo, ${ }^{90}$ M. Ramirez-Garcia, ${ }^{90}$ 
G. Ramirez-Sanchez,$^{90}$ R. Reyes-Almanza, ${ }^{90}$ A. Sanchez-Hernandez ${ }^{90}$ S. Carrillo Moreno, ${ }^{91}$ C. Oropeza Barrera, ${ }^{91}$ F. Vazquez Valencia,${ }^{91}$ J. Eysermans,${ }^{92}$ I. Pedraza,${ }^{92}$ H. A. Salazar Ibarguen,${ }^{92}$ C. Uribe Estrada,${ }^{92}$ A. Morelos Pineda,${ }^{93}$ D. Krofcheck, ${ }^{94}$ S. Bheesette, ${ }^{95}$ P. H. Butler, ${ }^{95}$ A. Ahmad, ${ }^{96}$ M. Ahmad, ${ }^{96}$ M. I. Asghar,${ }^{96}$ Q. Hassan, ${ }^{96}$ H. R. Hoorani ${ }^{96}$ A. Saddique, ${ }^{96}$ M. A. Shah, ${ }^{96}$ M. Shoaib,${ }^{96}$ M. Waqas, ${ }^{96}$ H. Bialkowska, ${ }^{97}$ M. Bluj, ${ }^{97}$ B. Boimska, ${ }^{97}$ T. Frueboes,${ }^{97}$ M. Górski, ${ }^{97}$ M. Kazana, ${ }^{97}$ M. Szleper, ${ }^{97}$ P. Traczyk,${ }^{97}$ P. Zalewski, ${ }^{97}$ K. Bunkowski, ${ }^{98}$ A. Byszuk,,${ }^{98, j}$ K. Doroba,${ }^{98}$ A. Kalinowski, ${ }^{98}$ M. Konecki, ${ }^{98}$ J. Krolikowski, ${ }^{98}$ M. Misiura, ${ }^{98}$ M. Olszewski, ${ }^{98}$ A. Pyskir,${ }^{98}$ M. Walczak, ${ }^{98}$ M. Araujo, ${ }^{99}$ P. Bargassa, ${ }^{99}$ C. Beirão Da Cruz E Silva, ${ }^{99}$ A. Di Francesco, ${ }^{99}$ P. Faccioli,${ }^{99}$ B. Galinhas, ${ }^{99}$ M. Gallinaro, ${ }^{99}$ J. Hollar, ${ }^{99}$ N. Leonardo, ${ }^{99}$ M. V. Nemallapudi, ${ }^{99}$ J. Seixas, ${ }^{99}$ G. Strong,${ }^{99}$ O. Toldaiev,${ }^{99}$ D. Vadruccio, ${ }^{99}$ J. Varela, ${ }^{99}$ S. Afanasiev,,${ }^{100}$ P. Bunin, ${ }^{100}$ M. Gavrilenko, ${ }^{100}$ I. Golutvin, ${ }^{100}$ I. Gorbunov, ${ }^{100}$ A. Kamenev, ${ }^{100}$ V. Karjavine, ${ }^{100}$ A. Lanev, ${ }^{100}$ A. Malakhov, ${ }^{100}$ V. Matveev, ${ }^{100, k k, 11}$ P. Moisenz, ${ }^{100}$ V. Palichik, ${ }^{100}$ V. Perelygin, ${ }^{100}$ S. Shmatov, ${ }^{100}$ S. Shulha, ${ }^{100}$ N. Skatchkov,${ }^{100}$ V. Smirnov ${ }^{100}$ N. Voytishin, ${ }^{100}$ A. Zarubin, ${ }^{100}$ V. Golovtsov, ${ }^{101}$ Y. Ivanov, ${ }^{101}$ V. Kim, ${ }^{101, m m}$ E. Kuznetsova, ${ }^{101, n n}$ P. Levchenko, ${ }^{101}$ V. Murzin, ${ }^{101}$ V. Oreshkin, ${ }^{101}$ I. Smirnov, ${ }^{101}$ D. Sosnov, ${ }^{101}$ V. Sulimov, ${ }^{101}$ L. Uvarov, ${ }^{101}$ S. Vavilov, ${ }^{101}$ A. Vorobyev, ${ }^{101}$ Yu. Andreev, ${ }^{102}$ A. Dermenev, ${ }^{102}$ S. Gninenko, ${ }^{102}$ N. Golubev, ${ }^{102}$ A. Karneyeu, ${ }^{102}$ M. Kirsanov, ${ }^{102}$

N. Krasnikov, ${ }^{102}$ A. Pashenkov, ${ }^{102}$ D. Tlisov, ${ }^{102}$ A. Toropin, ${ }^{102}$ V. Epshteyn, ${ }^{103}$ V. Gavrilov, ${ }^{103}$ N. Lychkovskaya, ${ }^{103}$ V. Popov, ${ }^{103}$ I. Pozdnyakov, ${ }^{103}$ G. Safronov, ${ }^{103}$ A. Spiridonov, ${ }^{103}$ A. Stepennov, ${ }^{103}$ V. Stolin, ${ }^{103}$ M. Toms, ${ }^{103}$ E. Vlasov, ${ }^{103}$ A. Zhokin, ${ }^{103}$ T. Aushev, ${ }^{104}$ R. Chistov, ${ }^{105,00}$ M. Danilov, ${ }^{105,00}$ P. Parygin, ${ }^{105}$ D. Philippov, ${ }^{105}$ S. Polikarpov, ${ }^{105,00}$

E. Tarkovskii, ${ }^{105}$ V. Andreev, ${ }^{106}$ M. Azarkin, ${ }^{106}$ I. Dremin,,${ }^{106,11}$ M. Kirakosyan, ${ }^{106}$ S. V. Rusakov, ${ }^{106}$ A. Terkulov, ${ }^{106}$ A. Baskakov, ${ }^{107}$ A. Belyaev ${ }^{107}$ E. Boos,${ }^{107}$ V. Bunichev,${ }^{107}$ M. Dubinin,,${ }^{107, p p}$ L. Dudko, ${ }^{107}$ V. Klyukhin, ${ }^{107}$ O. Kodolova, ${ }^{107}$ N. Korneeva, ${ }^{107}$ I. Lokhtin, ${ }^{107}$ I. Miagkov, ${ }^{107}$ S. Obraztsov, ${ }^{107}$ M. Perfilov, ${ }^{107}$ V. Savrin, ${ }^{107}$ P. Volkov, ${ }^{107}$ A. Barnyakov, ${ }^{108, q q}$ V. Blinov, ${ }^{108, q q}$ T. Dimova, ${ }^{108, q q}$ L. Kardapoltsev, ${ }^{108, q q}$ Y. Skovpen, ${ }^{108, q q}$ I. Azhgirey, ${ }^{109}$ I. Bayshev, ${ }^{109}$ S. Bitioukov, ${ }^{109}$

D. Elumakhov, ${ }^{109}$ A. Godizov, ${ }^{109}$ V. Kachanov, ${ }^{109}$ A. Kalinin, ${ }^{109}$ D. Konstantinov, ${ }^{109}$ P. Mandrik, ${ }^{109}$ V. Petrov, ${ }^{109}$ R. Ryutin, ${ }^{109}$ S. Slabospitskii, ${ }^{109}$ A. Sobol, ${ }^{109}$ S. Troshin,${ }^{109}$ N. Tyurin, ${ }^{109}$ A. Uzunian, ${ }^{109}$ A. Volkov, ${ }^{109}$ A. Babaev, ${ }^{110}$ S. Baidali, ${ }^{110}$ V. Okhotnikov, ${ }^{110}$ P. Adzic, ${ }^{11, \text { rr }}$ P. Cirkovic, ${ }^{111}$ D. Devetak, ${ }^{111}$ M. Dordevic, ${ }^{111}$ J. Milosevic, ${ }^{111}$ J. Alcaraz Maestre, ${ }^{112}$ A. Álvarez Fernández, ${ }^{112}$ I. Bachiller, ${ }^{112}$ M. Barrio Luna, ${ }^{112}$ J. A. Brochero Cifuentes, ${ }^{112}$ M. Cerrada, ${ }^{112}$ N. Colino, ${ }^{112}$ B. De La Cruz, ${ }^{112}$ A. Delgado Peris, ${ }^{112}$ C. Fernandez Bedoya, ${ }^{112}$ J. P. Fernández Ramos, ${ }^{112}$ J. Flix, ${ }^{112}$ M. C. Fouz, ${ }^{112}$ O. Gonzalez Lopez, ${ }^{112}$ S. Goy Lopez, ${ }^{112}$ J. M. Hernandez, ${ }^{112}$ M. I. Josa, ${ }^{112}$ D. Moran, ${ }^{112}$ A. Pérez-Calero Yzquierdo, ${ }^{112}$ J. Puerta Pelayo, ${ }^{112}$ I. Redondo, ${ }^{112}$ L. Romero, ${ }^{112}$ M. S. Soares, ${ }^{112}$ A. Triossi, ${ }^{112}$ C. Albajar, ${ }^{113}$ J. F. de Trocóniz, ${ }^{113}$ J. Cuevas, ${ }^{114}$ C. Erice, ${ }^{114}$ J. Fernandez Menendez, ${ }^{114}$ S. Folgueras, ${ }^{114}$ I. Gonzalez Caballero, ${ }^{114}$ J. R. González Fernández, ${ }^{114}$ E. Palencia Cortezon, ${ }^{114}$ V. Rodríguez Bouza, ${ }^{114}$ S. Sanchez Cruz, ${ }^{114}$ P. Vischia, ${ }^{114}$ J. M. Vizan Garcia, ${ }^{114}$ I. J. Cabrillo, ${ }^{115}$ A. Calderon, ${ }^{115}$ B. Chazin Quero, ${ }^{115}$ J. Duarte Campderros, ${ }^{115}$ M. Fernandez, ${ }^{115}$ P. J. Fernández Manteca, ${ }^{115}$ A. García Alonso, ${ }^{115}$ J. Garcia-Ferrero, ${ }^{115}$ G. Gomez, ${ }^{115}$ A. Lopez Virto, ${ }^{115}$ J. Marco, ${ }^{115}$ C. Martinez Rivero, ${ }^{115}$ P. Martinez Ruiz del Arbol, ${ }^{115}$ F. Matorras, ${ }^{115}$ J. Piedra Gomez, ${ }^{115}$ C. Prieels, ${ }^{115}$ T. Rodrigo, ${ }^{115}$ A. Ruiz-Jimeno, ${ }^{115}$ L. Scodellaro, ${ }^{115}$ N. Trevisani, ${ }^{115}$ I. Vila, ${ }^{115}$ R. Vilar Cortabitarte, ${ }^{115}$ N. Wickramage, ${ }^{116}$ D. Abbaneo, ${ }^{117}$ B. Akgun, ${ }^{117}$ E. Auffray, ${ }^{117}$ G. Auzinger, ${ }^{117}$ P. Baillon, ${ }^{117}$ A. H. Ball, ${ }^{117}$ D. Barney, ${ }^{117}$ J. Bendavid, ${ }^{117}$ M. Bianco, ${ }^{117}$ A. Bocci, ${ }^{117}$ C. Botta, ${ }^{117}$ E. Brondolin, ${ }^{117}$ T. Camporesi, ${ }^{117}$ M. Cepeda, ${ }^{117}$ G. Cerminara,${ }^{117}$ E. Chapon, ${ }^{117}$ Y. Chen, ${ }^{117}$ G. Cucciati, ${ }^{117}$ D. d'Enterria, ${ }^{117}$ A. Dabrowski, ${ }^{117}$ N. Daci,${ }^{117}$ V. Daponte, ${ }^{117}$ A. David, ${ }^{117}$ A. De Roeck,,${ }^{117}$ N. Deelen, ${ }^{117}$ M. Dobson, ${ }^{117}$ M. Dünser, ${ }^{117}$ N. Dupont,${ }^{117}$ A. Elliott-Peisert,${ }^{117}$ P. Everaerts, ${ }^{117}$ F. Fallavollita, ${ }^{117, \text { ss }}$ D. Fasanella, ${ }^{117}$ G. Franzoni, ${ }^{117}$ J. Fulcher, ${ }^{117}$ W. Funk, ${ }^{117}$ D. Gigi, ${ }^{117}$ A. Gilbert, ${ }^{117}$ K. Gill, ${ }^{117}$ F. Glege, ${ }^{117}$ M. Guilbaud, ${ }^{117}$ D. Gulhan, ${ }^{117}$ J. Hegeman, ${ }^{117}$ C. Heidegger,${ }^{117}$ V. Innocente,${ }^{117}$ A. Jafari, ${ }^{117}$ P. Janot, ${ }^{117}$ O. Karacheban, ${ }^{117, u}$ J. Kieseler, ${ }^{117}$ A. Kornmayer, ${ }^{117}$ M. Krammer, ${ }^{117, b}$ C. Lange,${ }^{117}$ P. Lecoq,${ }^{117}$ C. Lourenço, ${ }^{117}$ L. Malgeri, ${ }^{117}$ M. Mannelli, ${ }^{117}$ F. Meijers, ${ }^{117}$ J. A. Merlin, ${ }^{117}$ S. Mersi, ${ }^{117}$ E. Meschi, ${ }^{117}$ P. Milenovic, ${ }^{117, t t}$ F. Moortgat,${ }^{117}$ M. Mulders, ${ }^{117}$ J. Ngadiuba, ${ }^{117}$ S. Nourbakhsh ${ }^{117}$ S. Orfanelli, ${ }^{117}$ L. Orsini,${ }^{117}$ F. Pantaleo, ${ }^{117, \mathrm{r}}$ L. Pape, ${ }^{117}$ E. Perez, ${ }^{117}$ M. Peruzzi, ${ }^{117}$ A. Petrilli, ${ }^{117}$ G. Petrucciani, ${ }^{117}$ A. Pfeiffer, ${ }^{117}$ M. Pierini, ${ }^{117}$ F. M. Pitters,${ }^{117}$ D. Rabady, ${ }^{117}$ A. Racz, ${ }^{117}$ T. Reis,${ }^{117}$ G. Rolandi, ${ }^{117}$,uu M. Rovere ${ }^{117}$ H. Sakulin, ${ }^{117}$ C. Schäfer,${ }^{117}$ C. Schwick,${ }^{117}$ M. Seidel,${ }^{117}$ M. Selvaggi, ${ }^{117}$ A. Sharma, ${ }^{117}$ P. Silva, ${ }^{117}$ P. Sphicas, ${ }^{117, \text { vv }}$ A. Stakia, ${ }^{117}$ J. Steggemann, ${ }^{117}$ M. Tosi, ${ }^{117}$ D. Treille, ${ }^{117}$ A. Tsirou, ${ }^{117}$ V. Veckalns, ${ }^{117, w w}$ M. Verzetti, ${ }^{117}$ W. D. Zeuner, ${ }^{117}$ L. Caminada, ${ }^{118, x x}$ K. Deiters, ${ }^{118}$ W. Erdmann, ${ }^{118}$ R. Horisberger, ${ }^{118}$ Q. Ingram, ${ }^{118}$ H. C. Kaestli, ${ }^{118}$

D. Kotlinski, ${ }^{118}$ U. Langenegger, ${ }^{118}$ T. Rohe, ${ }^{118}$ S. A. Wiederkehr, ${ }^{118}$ M. Backhaus, ${ }^{119}$ L. Bäni, ${ }^{119}$ P. Berger,${ }^{119}$

N. Chernyavskaya, ${ }^{119}$ G. Dissertori, ${ }^{119}$ M. Dittmar, ${ }^{119}$ M. Donegà, ${ }^{119}$ C. Dorfer ${ }^{119}$ T. A. Gómez Espinosa, ${ }^{119}$ C. Grab, ${ }^{119}$ D. Hits, ${ }^{119}$ T. Klijnsma, ${ }^{119}$ W. Lustermann, ${ }^{119}$ R. A. Manzoni, ${ }^{119}$ M. Marionneau, ${ }^{119}$ M. T. Meinhard, ${ }^{119}$ F. Micheli, ${ }^{119}$ 
P. Musella, ${ }^{119}$ F. Nessi-Tedaldi, ${ }^{119}$ J. Pata, ${ }^{119}$ F. Pauss, ${ }^{119}$ G. Perrin, ${ }^{119}$ L. Perrozzi, ${ }^{119}$ S. Pigazzini, ${ }^{119}$ M. Quittnat, ${ }^{119}$ C. Reissel, ${ }^{119}$ D. Ruini, ${ }^{119}$ D. A. Sanz Becerra, ${ }^{119}$ M. Schönenberger, ${ }^{119}$ L. Shchutska, ${ }^{119}$ V. R. Tavolaro, ${ }^{119}$

K. Theofilatos, ${ }^{119}$ M. L. Vesterbacka Olsson, ${ }^{119}$ R. Wallny, ${ }^{119}$ D. H. Zhu, ${ }^{119}$ T. K. Aarrestad, ${ }^{120}$ C. Amsler, ${ }^{120, y y}$ D. Brzhechko, ${ }^{120}$ M. F. Canelli, ${ }^{120}$ A. De Cosa, ${ }^{120}$ R. Del Burgo, ${ }^{120}$ S. Donato, ${ }^{120}$ C. Galloni, ${ }^{120}$ T. Hreus, ${ }^{120}$ B. Kilminster, ${ }^{120}$ S. Leontsinis, ${ }^{120}$ I. Neutelings, ${ }^{120}$ D. Pinna, ${ }^{120}$ G. Rauco, ${ }^{120}$ P. Robmann, ${ }^{120}$ D. Salerno, ${ }^{120}$ K. Schweiger, ${ }^{120}$ C. Seitz, ${ }^{120}$ Y. Takahashi, ${ }^{120}$ A. Zucchetta, ${ }^{120}$ Y. H. Chang, ${ }^{121}$ K. y. Cheng, ${ }^{121}$ T. H. Doan, ${ }^{121}$ R. Khurana, ${ }^{121}$ C. M. Kuo, ${ }^{121}$ W. Lin, ${ }^{121}$ A. Pozdnyakov, ${ }^{121}$ S. S. Yu, ${ }^{121}$ P. Chang, ${ }^{122}$ Y. Chao, ${ }^{122}$ K. F. Chen, ${ }^{122}$ P. H. Chen, ${ }^{122}$ W.-S. Hou, ${ }^{122}$ Arun Kumar, ${ }^{122}$ Y. F. Liu, ${ }^{122}$ R.-S. Lu, ${ }^{122}$ E. Paganis, ${ }^{122}$ A. Psallidas, ${ }^{122}$ A. Steen, ${ }^{122}$ B. Asavapibhop, ${ }^{123}$ N. Srimanobhas, ${ }^{123}$ N. Suwonjandee, ${ }^{123}$ A. Bat, ${ }^{124}$ F. Boran, ${ }^{124}$ S. Cerci, ${ }^{124, z z}$ S. Damarseckin, ${ }^{124}$ Z. S. Demiroglu, ${ }^{124}$ F. Dolek, ${ }^{124}$ C. Dozen, ${ }^{124}$ I. Dumanoglu, ${ }^{124}$ S. Girgis, ${ }^{124}$ G. Gokbulut, ${ }^{124}$ Y. Guler, ${ }^{124}$ E. Gurpinar, ${ }^{124}$ I. Hos, ${ }^{124, \text { aaa }}$ C. Isik, ${ }^{124}$ E. E. Kangal, ${ }^{124, \text { bbb }}$

O. Kara, ${ }^{124}$ A. Kayis Topaksu, ${ }^{124}$ U. Kiminsu, ${ }^{124}$ M. Oglakci, ${ }^{124}$ G. Onengut, ${ }^{124}$ K. Ozdemir, ${ }^{124, c c c}$ S. Ozturk, ${ }^{124, d d d}$ D. Sunar Cerci, ${ }^{124, z z}$ B. Tali, ${ }^{124, z z}$ U. G. Tok, ${ }^{124}$ S. Turkcapar, ${ }^{124}$ I. S. Zorbakir, ${ }^{124}$ C. Zorbilmez, ${ }^{124}$ B. Isildak, ${ }^{125, \text { eee }}$ G. Karapinar, ${ }^{125, \text { fff }}$ M. Yalvac, ${ }^{125}$ M. Zeyrek, ${ }^{125}$ I. O. Atakisi, ${ }^{126}$ E. Gülmez, ${ }^{126}$ M. Kaya, ${ }^{126, g g g}$ O. Kaya, ${ }^{126, h h h}$ S. Ozkorucuklu, ${ }^{126, i i i}$ S. Tekten, ${ }^{126}$ E. A. Yetkin, ${ }^{126, j j j}$ M. N. Agaras, ${ }^{127}$ A. Cakir, ${ }^{127}$ K. Cankocak, ${ }^{127}$ Y. Komurcu, ${ }^{127}$ S. Sen, ${ }^{127, k k k}$ B. Grynyov, ${ }^{128}$ L. Levchuk, ${ }^{129}$ F. Ball, ${ }^{130}$ L. Beck, ${ }^{130}$ J. J. Brooke, ${ }^{130}$ D. Burns, ${ }^{130}$ E. Clement,,${ }^{130}$ D. Cussans, ${ }^{130}$ O. Davignon, ${ }^{130}$ H. Flacher, ${ }^{130}$ J. Goldstein, ${ }^{130}$ G. P. Heath, ${ }^{130}$ H. F. Heath, ${ }^{130}$ L. Kreczko, ${ }^{130}$ D. M. Newbold, ${ }^{130,111}$ S. Paramesvaran, ${ }^{130}$ B. Penning, ${ }^{130}$ T. Sakuma, ${ }^{130}$ D. Smith, ${ }^{130}$ V. J. Smith, ${ }^{130}$ J. Taylor, ${ }^{130}$ A. Titterton, ${ }^{130}$ K. W. Bell, ${ }^{131}$ A. Belyaev, ${ }^{131, \mathrm{mmm}}$ C. Brew, ${ }^{131}$ R. M. Brown, ${ }^{131}$ D. Cieri, ${ }^{131}$ D. J. A. Cockerill, ${ }^{131}$ J. A. Coughlan, ${ }^{131}$ K. Harder, ${ }^{131}$ S. Harper ${ }^{131}$ J. Linacre, ${ }^{131}$ E. Olaiya, ${ }^{131}$ D. Petyt,${ }^{131}$ C. H. Shepherd-Themistocleous, ${ }^{131}$ A. Thea,${ }^{131}$ I. R. Tomalin, ${ }^{131}$

T. Williams, ${ }^{131}$ W. J. Womersley, ${ }^{131}$ R. Bainbridge, ${ }^{132}$ P. Bloch, ${ }^{132}$ J. Borg, ${ }^{132}$ S. Breeze, ${ }^{132}$ O. Buchmuller, ${ }^{132}$ A. Bundock, ${ }^{132}$ D. Colling, ${ }^{132}$ P. Dauncey, ${ }^{132}$ G. Davies, ${ }^{132}$ M. Della Negra, ${ }^{132}$ R. Di Maria, ${ }^{132}$ Y. Haddad, ${ }^{132}$ G. Hall, ${ }^{132}$ G. Iles, ${ }^{132}$ T. James ${ }^{132}$ M. Komm, ${ }^{132}$ C. Laner, ${ }^{132}$ L. Lyons, ${ }^{132}$ A.-M. Magnan, ${ }^{132}$ S. Malik, ${ }^{132}$ A. Martelli, ${ }^{132}$ J. Nash, ${ }^{132, n n n}$ A. Nikitenko, ${ }^{132, \mathrm{~h}}$ V. Palladino, ${ }^{132}$ M. Pesaresi, ${ }^{132}$ D. M. Raymond, ${ }^{132}$ A. Richards, ${ }^{132}$ A. Rose, ${ }^{132}$ E. Scott, ${ }^{132}$ C. Seez, ${ }^{132}$ A. Shtipliyski, ${ }^{132}$ G. Singh, ${ }^{132}$ M. Stoye,${ }^{132}$ T. Strebler ${ }^{132}$ S. Summers, ${ }^{132}$ A. Tapper ${ }^{132}$ K. Uchida ${ }^{132}$ T. Virdee, ${ }^{132, \mathrm{r}}$ N. Wardle, ${ }^{132}$ D. Winterbottom, ${ }^{132}$ J. Wright, ${ }^{132}$ S. C. Zenz, ${ }^{132}$ J. E. Cole ${ }^{133}$ P. R. Hobson, ${ }^{133}$ A. Khan, ${ }^{133}$ P. Kyberd, ${ }^{133}$ C. K. Mackay, ${ }^{133}$ A. Morton, ${ }^{133}$ I. D. Reid, ${ }^{133}$ L. Teodorescu, ${ }^{133}$ S. Zahid, ${ }^{133}$ K. Call, ${ }^{134}$ J. Dittmann, ${ }^{134}$ K. Hatakeyama, ${ }^{134}$ H. Liu, ${ }^{134}$ C. Madrid,${ }^{134}$ B. Mcmaster, ${ }^{134}$ N. Pastika, ${ }^{134}$ C. Smith, ${ }^{134}$ R. Bartek, ${ }^{135}$ A. Dominguez, ${ }^{135}$ A. Buccilli, ${ }^{136}$ S. I. Cooper, ${ }^{136}$ C. Henderson, ${ }^{136}$ P. Rumerio, ${ }^{136}$ C. West ${ }^{136}$ D. Arcaro, ${ }^{137}$ T. Bose, ${ }^{137}$ D. Gastler, ${ }^{137}$ D. Rankin, ${ }^{137}$ C. Richardson, ${ }^{137}$ J. Rohlf, ${ }^{137}$ L. Sulak, ${ }^{137}$ D. Zou, ${ }^{137}$ G. Benelli, ${ }^{138}$ X. Coubez, ${ }^{138}$ D. Cutts, ${ }^{138}$ M. Hadley, ${ }^{138}$ J. Hakala, ${ }^{138}$ U. Heintz, ${ }^{138}$ J. M. Hogan, ${ }^{138,000}$ K. H. M. Kwok, ${ }^{138}$ E. Laird, ${ }^{138}$ G. Landsberg, ${ }^{138}$ J. Lee, ${ }^{138}$ Z. Mao, ${ }^{138}$ M. Narain, ${ }^{138}$ S. Sagir, ${ }^{138, p p p}$ R. Syarif, ${ }^{138}$ E. Usai, ${ }^{138}$ D. Yu, ${ }^{138}$ R. Band, ${ }^{139}$ C. Brainerd, ${ }^{139}$ R. Breedon, ${ }^{139}$ D. Burns, ${ }^{139}$ M. Calderon De La Barca Sanchez, ${ }^{139}$ M. Chertok, ${ }^{139}$ J. Conway, ${ }^{139}$ R. Conway, ${ }^{139}$ P. T. Cox, ${ }^{139}$ R. Erbacher, ${ }^{139}$ C. Flores, ${ }^{139}$ G. Funk, ${ }^{139}$ W. Ko, ${ }^{139}$ O. Kukral, ${ }^{139}$ R. Lander ${ }^{139}$ M. Mulhearn, ${ }^{139}$ D. Pellett, ${ }^{139}$ J. Pilot, ${ }^{139}$ S. Shalhout, ${ }^{139}$ M. Shi, ${ }^{139}$ D. Stolp,${ }^{139}$ D. Taylor, ${ }^{139}$ K. Tos, ${ }^{139}$ M. Tripathi, ${ }^{139}$ Z. Wang, ${ }^{139}$ F. Zhang, ${ }^{139}$ M. Bachtis, ${ }^{140}$ C. Bravo, ${ }^{140}$ R. Cousins, ${ }^{140}$ A. Dasgupta, ${ }^{140}$ A. Florent, ${ }^{140}$ J. Hauser,${ }^{140}$ M. Ignatenko, ${ }^{140}$ N. Mccoll, ${ }^{140}$ S. Regnard, ${ }^{140}$ D. Saltzberg, ${ }^{140}$ C. Schnaible, ${ }^{140}$ V. Valuev, ${ }^{140}$ E. Bouvier, ${ }^{141}$ K. Burt, ${ }^{141}$ R. Clare,${ }^{141}$ J. W. Gary, ${ }^{141}$ S. M. A. Ghiasi Shirazi, ${ }^{141}$ G. Hanson, ${ }^{141}$ G. Karapostoli, ${ }^{141}$ E. Kennedy, ${ }^{141}$ F. Lacroix, ${ }^{141}$ O. R. Long, ${ }^{141}$ M. Olmedo Negrete, ${ }^{141}$ M. I. Paneva, ${ }^{141}$ W. Si, ${ }^{141}$ L. Wang, ${ }^{141}$ H. Wei, ${ }^{141}$ S. Wimpenny, ${ }^{141}$ B. R. Yates, ${ }^{141}$ J. G. Branson, ${ }^{142}$ P. Chang, ${ }^{142}$ S. Cittolin, ${ }^{142}$ M. Derdzinski, ${ }^{142}$ R. Gerosa, ${ }^{142}$ D. Gilbert, ${ }^{142}$ B. Hashemi, ${ }^{142}$ A. Holzner, ${ }^{142}$ D. Klein, ${ }^{142}$ G. Kole, ${ }^{142}$ V. Krutelyov, ${ }^{142}$ J. Letts, ${ }^{142}$ M. Masciovecchio, ${ }^{142}$ D. Olivito, ${ }^{142}$ S. Padhi, ${ }^{142}$ M. Pieri, ${ }^{142}$ M. Sani, ${ }^{142}$ V. Sharma, ${ }^{142}$ S. Simon, ${ }^{142}$ M. Tadel,${ }^{142}$ A. Vartak, ${ }^{142}$ S. Wasserbaech, ${ }^{142, q q 9}$ J. Wood, ${ }^{142}$ F. Würthwein, ${ }^{142}$ A. Yagil, ${ }^{142}$ G. Zevi Della Porta, ${ }^{142}$ N. Amin, ${ }^{143}$ R. Bhandari, ${ }^{143}$ J. Bradmiller-Feld, ${ }^{143}$ C. Campagnari, ${ }^{143}$ M. Citron, ${ }^{143}$ A. Dishaw, ${ }^{143}$ V. Dutta, ${ }^{143}$ M. Franco Sevilla, ${ }^{143}$ L. Gouskos,${ }^{143}$ R. Heller, ${ }^{143}$ J. Incandela ${ }^{143}$ A. Ovcharova, ${ }^{143}$ H. Qu, ${ }^{143}$ J. Richman, ${ }^{143}$ D. Stuart, ${ }^{143}$ I. Suarez, ${ }^{143}$ S. Wang, ${ }^{143}$ J. Yoo, ${ }^{143}$ D. Anderson, ${ }^{144}$ A. Bornheim, ${ }^{144}$ J. M. Lawhorn, ${ }^{144}$ H. B. Newman, ${ }^{144}$ T. Q. Nguyen, ${ }^{144}$ M. Spiropulu, ${ }^{144}$ J. R. Vlimant, ${ }^{144}$ R. Wilkinson, ${ }^{144}$ S. Xie, ${ }^{144}$ Z. Zhang, ${ }^{144}$ R. Y. Zhu, ${ }^{144}$ M. B. Andrews, ${ }^{145}$ T. Ferguson, ${ }^{145}$ T. Mudholkar, ${ }^{145}$ M. Paulini, ${ }^{145}$ M. Sun, ${ }^{145}$ I. Vorobiev, ${ }^{145}$ M. Weinberg, ${ }^{145}$ J. P. Cumalat, ${ }^{146}$ W. T. Ford, ${ }^{146}$ F. Jensen, ${ }^{146}$ A. Johnson, ${ }^{146}$ M. Krohn, ${ }^{146}$ E. MacDonald, ${ }^{146}$ T. Mulholland, ${ }^{146}$ R. Patel, ${ }^{146}$ K. Stenson, ${ }^{146}$ K. A. Ulmer, ${ }^{146}$ S. R. Wagner,${ }^{146}$ J. Alexander, ${ }^{147}$ J. Chaves, ${ }^{147}$ Y. Cheng, ${ }^{147}$ J. Chu, ${ }^{147}$ A. Datta, ${ }^{147}$ K. Mcdermott, ${ }^{147}$ N. Mirman, ${ }^{147}$ J. R. Patterson, ${ }^{147}$ D. Quach, ${ }^{147}$ A. Rinkevicius, ${ }^{147}$ A. Ryd ${ }^{147}$ L. Skinnari, ${ }^{147}$ L. Soffi, ${ }^{147}$ S. M. Tan, ${ }^{147}$ Z. Tao, ${ }^{147}$ J. Thom, ${ }^{147}$ J. Tucker, ${ }^{147}$ 
P. Wittich, ${ }^{147}$ M. Zientek, ${ }^{147}$ S. Abdullin, ${ }^{148}$ M. Albrow, ${ }^{148}$ M. Alyari, ${ }^{148}$ G. Apollinari, ${ }^{148}$ A. Apresyan, ${ }^{148}$ A. Apyan, ${ }^{148}$ S. Banerjee ${ }^{148}$ L. A. T. Bauerdick, ${ }^{148}$ A. Beretvas, ${ }^{148}$ J. Berryhill, ${ }^{148}$ P. C. Bhat,${ }^{148}$ G. Bolla, ${ }^{148, a}$ K. Burkett, ${ }^{148}$ J. N. Butler, ${ }^{148}$ A. Canepa, ${ }^{148}$ G. B. Cerati, ${ }^{148}$ H. W. K. Cheung, ${ }^{148}$ F. Chlebana, ${ }^{148}$ M. Cremonesi, ${ }^{148}$ J. Duarte, ${ }^{148}$ V. D. Elvira, ${ }^{148}$ J. Freeman, ${ }^{148}$ Z. Gecse, ${ }^{148}$ E. Gottschalk, ${ }^{148}$ L. Gray,${ }^{148}$ D. Green, ${ }^{148}$ S. Grünendahl,${ }^{148}$ O. Gutsche, ${ }^{148}$ J. Hanlon, ${ }^{148}$ R. M. Harris, ${ }^{148}$ S. Hasegawa, ${ }^{148}$ J. Hirschauer, ${ }^{148}$ Z. Hu, ${ }^{148}$ B. Jayatilaka, ${ }^{148}$ S. Jindariani, ${ }^{148}$ M. Johnson, ${ }^{148}$ U. Joshi, ${ }^{148}$ B. Klima,${ }^{148}$ M. J. Kortelainen, ${ }^{148}$ B. Kreis, ${ }^{148}$ S. Lammel, ${ }^{148}$ D. Lincoln, ${ }^{148}$ R. Lipton, ${ }^{148}$ M. Liu, ${ }^{148}$ T. Liu, ${ }^{148}$ J. Lykken, ${ }^{148}$ K. Maeshima, ${ }^{148}$ J. M. Marraffino, ${ }^{148}$ D. Mason, ${ }^{148}$ P. McBride,${ }^{148}$ P. Merkel, ${ }^{148}$ S. Mrenna, ${ }^{148}$ S. Nahn, ${ }^{148}$ V. O’Dell, ${ }^{148}$ K. Pedro,${ }^{148}$ C. Pena, ${ }^{148}$ O. Prokofyev, ${ }^{148}$ G. Rakness, ${ }^{148}$ L. Ristori, ${ }^{148}$ A. Savoy-Navarro, ${ }^{148, r r r}$ B. Schneider, ${ }^{148}$ E. Sexton-Kennedy, ${ }^{148}$ A. Soha, ${ }^{148}$ W. J. Spalding, ${ }^{148}$ L. Spiegel, ${ }^{148}$ S. Stoynev ${ }^{148}$ J. Strait, ${ }^{148}$ N. Strobbe,${ }^{148}$ L. Taylor, ${ }^{148}$ S. Tkaczyk, ${ }^{148}$ N. V. Tran, ${ }^{148}$ L. Uplegger,${ }^{148}$ E. W. Vaandering, ${ }^{148}$ C. Vernieri, ${ }^{148}$ M. Verzocchi, ${ }^{148}$ R. Vidal, ${ }^{148}$ M. Wang, ${ }^{148}$ H. A. Weber, ${ }^{148}$ A. Whitbeck, ${ }^{148}$ D. Acosta, ${ }^{149}$ P. Avery, ${ }^{149}$ P. Bortignon, ${ }^{149}$ D. Bourilkov, ${ }^{149}$ A. Brinkerhoff, ${ }^{149}$ L. Cadamuro, ${ }^{149}$ A. Carnes, ${ }^{149}$ M. Carver, ${ }^{149}$ D. Curry, ${ }^{149}$ R. D. Field, ${ }^{149}$ S. V. Gleyzer, ${ }^{149}$ B. M. Joshi, ${ }^{149}$ J. Konigsberg, ${ }^{149}$ A. Korytov, ${ }^{149}$ K. H. Lo, ${ }^{149}$ P. Ma, ${ }^{149}$ K. Matchev, ${ }^{149}$ H. Mei,${ }^{149}$ G. Mitselmakher, ${ }^{149}$ D. Rosenzweig, ${ }^{149}$ K. Shi, ${ }^{149}$ D. Sperka, ${ }^{149}$ J. Wang, ${ }^{149}$ S. Wang, ${ }^{149}$ X. Zuo, ${ }^{149}$ Y. R. Joshi, ${ }^{150}$ S. Linn, ${ }^{150}$ A. Ackert, ${ }^{151}$ T. Adams, ${ }^{151}$ A. Askew, ${ }^{151}$ S. Hagopian, ${ }^{151}$ V. Hagopian, ${ }^{151}$ K. F. Johnson, ${ }^{151}$ T. Kolberg, ${ }^{151}$ G. Martinez, ${ }^{151}$ T. Perry, ${ }^{151}$ H. Prosper, ${ }^{151}$ A. Saha, ${ }^{151}$ C. Schiber, ${ }^{151}$ R. Yohay, ${ }^{151}$ M. M. Baarmand, ${ }^{152}$ V. Bhopatkar, ${ }^{152}$ S. Colafranceschi, ${ }^{152}$ M. Hohlmann, ${ }^{152}$ D. Noonan, ${ }^{152}$ M. Rahmani ${ }^{152}$ T. Roy, ${ }^{152}$ F. Yumiceva, ${ }^{152}$ M. R. Adams,${ }^{153}$ L. Apanasevich, ${ }^{153}$ D. Berry, ${ }^{153}$ R. R. Betts, ${ }^{153}$ R. Cavanaugh, ${ }^{153}$ X. Chen, ${ }^{153}$ S. Dittmer, ${ }^{153}$ O. Evdokimov,${ }^{153}$ C. E. Gerber, ${ }^{153}$ D. A. Hangal, ${ }^{153}$ D. J. Hofman, ${ }^{153}$ K. Jung, ${ }^{153}$ J. Kamin,${ }^{153}$ C. Mills, ${ }^{153}$ I. D. Sandoval Gonzalez, ${ }^{153}$ M. B. Tonjes, ${ }^{153}$ H. Trauger, ${ }^{153}$ N. Varelas, ${ }^{153}$ H. Wang, ${ }^{153}$ X. Wang, ${ }^{153}$ Z. Wu, ${ }^{153}$ J. Zhang, ${ }^{153}$ M. Alhusseini, ${ }^{154}$ B. Bilki, ${ }^{154, s s s}$ W. Clarida,${ }^{154}$ K. Dilsiz, ${ }^{154, t t t}$ S. Durgut, ${ }^{154}$ R. P. Gandrajula, ${ }^{154}$ M. Haytmyradov, ${ }^{154}$ V. Khristenko, ${ }^{154}$ J.-P. Merlo ${ }^{154}$ A. Mestvirishvili, ${ }^{154}$ A. Moeller, ${ }^{154}$ J. Nachtman, ${ }^{154}$ H. Ogul, ${ }^{154, \text { uuu }}$ Y. Onel, ${ }^{154}$ F. Ozok,,${ }^{154, \text { vvv }}$ A. Penzo, ${ }^{154}$ C. Snyder,${ }^{154}$ E. Tiras, ${ }^{154}$ J. Wetzel, ${ }^{154}$ B. Blumenfeld, ${ }^{155}$ A. Cocoros, ${ }^{155}$ N. Eminizer, ${ }^{155}$ D. Fehling, ${ }^{155}$ L. Feng,${ }^{155}$ A. V. Gritsan, ${ }^{155}$ W. T. Hung, ${ }^{155}$ P. Maksimovic, ${ }^{155}$ J. Roskes, ${ }^{155}$ U. Sarica, ${ }^{155}$ M. Swartz, ${ }^{155}$ M. Xiao, ${ }^{155}$ C. You, ${ }^{155}$ A. Al-bataineh, ${ }^{156}$ P. Baringer, ${ }^{156}$ A. Bean, ${ }^{156}$ S. Boren, ${ }^{156}$ J. Bowen, ${ }^{156}$ A. Bylinkin, ${ }^{156}$ J. Castle, ${ }^{156}$ S. Khalil, ${ }^{156}$ A. Kropivnitskaya, ${ }^{156}$ D. Majumder, ${ }^{156}$ W. Mcbrayer ${ }^{156}$ M. Murray, ${ }^{156}$ C. Rogan, ${ }^{156}$ S. Sanders, ${ }^{156}$ E. Schmitz, ${ }^{156}$ J. D. Tapia Takaki, ${ }^{156}$ Q. Wang, ${ }^{156}$ S. Duric, ${ }^{157}$ A. Ivanov,${ }^{157}$ K. Kaadze, ${ }^{157}$ D. Kim, ${ }^{157}$ Y. Maravin, ${ }^{157}$ D. R. Mendis, ${ }^{157}$ T. Mitchell, ${ }^{157}$ A. Modak, ${ }^{157}$ A. Mohammadi, ${ }^{157}$ L. K. Saini, ${ }^{157}$ N. Skhirtladze,${ }^{157}$ F. Rebassoo, ${ }^{158}$ D. Wright, ${ }^{158}$ A. Baden, ${ }^{159}$ O. Baron, ${ }^{159}$ A. Belloni, ${ }^{159}$ S. C. Eno, ${ }^{159}$ Y. Feng, ${ }^{159}$ C. Ferraioli, ${ }^{159}$ N. J. Hadley, ${ }^{159}$ S. Jabeen, ${ }^{159}$ G. Y. Jeng, ${ }^{159}$ R. G. Kellogg, ${ }^{159}$ J. Kunkle, ${ }^{159}$ A. C. Mignerey, ${ }^{159}$ S. Nabili, ${ }^{159}$ F. Ricci-Tam, ${ }^{159}$ Y. H. Shin, ${ }^{159}$ A. Skuja, ${ }^{159}$ S. C. Tonwar ${ }^{159}$ K. Wong ${ }^{159}$ D. Abercrombie, ${ }^{160}$ B. Allen, ${ }^{160}$ V. Azzolini, ${ }^{160}$ A. Baty, ${ }^{160}$ G. Bauer, ${ }^{160}$ R. Bi, ${ }^{160}$ S. Brandt, ${ }^{160}$ W. Busza, ${ }^{160}$ I. A. Cali, ${ }^{160}$ M. D' Alfonso, ${ }^{160}$ Z. Demiragli, ${ }^{160}$ G. Gomez Ceballos, ${ }^{160}$ M. Goncharov, ${ }^{160}$ P. Harris, ${ }^{160}$ D. Hsu, ${ }^{160}$ M. Hu, ${ }^{160}$ Y. Iiyama, ${ }^{160}$ G. M. Innocenti, ${ }^{160}$ M. Klute, ${ }^{160}$ D. Kovalskyi, ${ }^{160}$ Y.-J. Lee, ${ }^{160}$ P. D. Luckey, ${ }^{160}$ B. Maier, ${ }^{160}$ A. C. Marini, ${ }^{160}$ C. Mcginn, ${ }^{160}$ C. Mironov, ${ }^{160}$ S. Narayanan, ${ }^{160}$ X. Niu, ${ }^{160}$ C. Paus, ${ }^{160}$ C. Roland, ${ }^{160}$ G. Roland, ${ }^{160}$ G. S. F. Stephans, ${ }^{160}$ K. Sumorok, ${ }^{160}$ K. Tatar, ${ }^{160}$ D. Velicanu, ${ }^{160}$ J. Wang, ${ }^{160}$ T. W. Wang, ${ }^{160}$ B. Wyslouch, ${ }^{160}$ S. Zhaozhong, ${ }^{160}$ A. C. Benvenuti,${ }^{161}$ R. M. Chatterjee, ${ }^{161}$ A. Evans, ${ }^{161}$

P. Hansen, ${ }^{161}$ Sh. Jain, ${ }^{161}$ S. Kalafut, ${ }^{161}$ Y. Kubota, ${ }^{161}$ Z. Lesko, ${ }^{161}$ J. Mans, ${ }^{161}$ N. Ruckstuhl, ${ }^{161}$ R. Rusack, ${ }^{161}$

J. Turkewitz, ${ }^{161}$ M. A. Wadud, ${ }^{161}$ J. G. Acosta, ${ }^{162}$ S. Oliveros, ${ }^{162}$ E. Avdeeva, ${ }^{163}$ K. Bloom,${ }^{163}$ D. R. Claes, ${ }^{163}$ C. Fangmeier, ${ }^{163}$ F. Golf, ${ }^{163}$ R. Gonzalez Suarez, ${ }^{163}$ R. Kamalieddin, ${ }^{163}$ I. Kravchenko, ${ }^{163}$ J. Monroy, ${ }^{163}$ J. E. Siado, ${ }^{163}$ G. R. Snow, ${ }^{163}$ B. Stieger, ${ }^{163}$ A. Godshalk, ${ }^{164}$ C. Harrington, ${ }^{164}$ I. Iashvili, ${ }^{164}$ A. Kharchilava, ${ }^{164}$ C. Mclean, ${ }^{164}$ D. Nguyen, ${ }^{164}$ A. Parker, ${ }^{164}$ S. Rappoccio, ${ }^{164}$ B. Roozbahani, ${ }^{164}$ G. Alverson, ${ }^{165}$ E. Barberis, ${ }^{165}$ C. Freer,${ }^{165}$ A. Hortiangtham, ${ }^{165}$ D. M. Morse, ${ }^{165}$ T. Orimoto, ${ }^{165}$ R. Teixeira De Lima, ${ }^{165}$ T. Wamorkar, ${ }^{165}$ B. Wang, ${ }^{165}$ A. Wisecarver, ${ }^{165}$ D. Wood, ${ }^{165}$ S. Bhattacharya, ${ }^{166}$ O. Charaf, ${ }^{166}$ K. A. Hahn, ${ }^{166}$ N. Mucia, ${ }^{166}$ N. Odell, ${ }^{166}$ M. H. Schmitt, ${ }^{166}$ K. Sung, ${ }^{166}$ M. Trovato, ${ }^{166}$ M. Velasco, ${ }^{166}$ R. Bucci, ${ }^{167}$ N. Dev ${ }^{167}$ M. Hildreth,${ }^{167}$ K. Hurtado Anampa,${ }^{167}$ C. Jessop,${ }^{167}$ D. J. Karmgard, ${ }^{167}$ N. Kellams, ${ }^{167}$ K. Lannon, ${ }^{167}$ W. Li,${ }^{167}$ N. Loukas, ${ }^{167}$ N. Marinelli, ${ }^{167}$ F. Meng, ${ }^{167}$ C. Mueller,${ }^{167}$ Y. Musienko, ${ }^{167, k k}$ M. Planer, ${ }^{167}$ A. Reinsvold ${ }^{167}$ R. Ruchti, ${ }^{167}$ P. Siddireddy, ${ }^{167}$ G. Smith, ${ }^{167}$ S. Taroni, ${ }^{167}$ M. Wayne, ${ }^{167}$ A. Wightman,${ }^{167}$ M. Wolf, ${ }^{167}$ A. Woodard, ${ }^{167}$ J. Alimena, ${ }^{168}$ L. Antonelli, ${ }^{168}$ B. Bylsma, ${ }^{168}$ L. S. Durkin,${ }^{168}$ S. Flowers, ${ }^{168}$ B. Francis, ${ }^{168}$ A. Hart, ${ }^{168}$ C. Hill, ${ }^{168}$ W. Ji, ${ }^{168}$ T. Y. Ling, ${ }^{168}$ W. Luo, ${ }^{168}$ B. L. Winer, ${ }^{168}$ S. Cooperstein, ${ }^{169}$ P. Elmer,${ }^{169}$ J. Hardenbrook, ${ }^{169}$ S. Higginbotham, ${ }^{169}$ A. Kalogeropoulos, ${ }^{169}$ D. Lange, ${ }^{169}$ M. T. Lucchini, ${ }^{169}$ J. Luo, ${ }^{169}$ D. Marlow, ${ }^{169}$ K. Mei, ${ }^{169}$ I. Ojalvo, ${ }^{169}$ J. Olsen, ${ }^{169}$ C. Palmer, ${ }^{169}$ P. Piroué, ${ }^{169}$ J. Salfeld-Nebgen, ${ }^{169}$ D. Stickland, ${ }^{169}$ C. Tully, ${ }^{169}$ S. Malik, ${ }^{170}$ S. Norberg, ${ }^{170}$ 
A. Barker, ${ }^{171}$ V. E. Barnes, ${ }^{171}$ S. Das, ${ }^{171}$ L. Gutay, ${ }^{171}$ M. Jones, ${ }^{171}$ A. W. Jung, ${ }^{171}$ A. Khatiwada, ${ }^{171}$ B. Mahakud ${ }^{171}$ D. H. Miller, ${ }^{171}$ N. Neumeister, ${ }^{171}$ C. C. Peng, ${ }^{171}$ S. Piperov, ${ }^{171}$ H. Qiu, ${ }^{171}$ J. F. Schulte, ${ }^{171}$ J. Sun, ${ }^{171}$ F. Wang, ${ }^{171}$ R. Xiao, ${ }^{171}$ W. Xie, ${ }^{171}$ T. Cheng, ${ }^{172}$ J. Dolen, ${ }^{172}$ N. Parashar,${ }^{172}$ Z. Chen, ${ }^{173}$ K. M. Ecklund,${ }^{173}$ S. Freed, ${ }^{173}$ F. J. M. Geurts, ${ }^{173}$ M. Kilpatrick, ${ }^{173}$ W. Li, ${ }^{173}$ B. P. Padley, ${ }^{173}$ R. Redjimi, ${ }^{173}$ J. Roberts, ${ }^{173}$ J. Rorie, ${ }^{173}$ W. Shi, ${ }^{173}$ Z. Tu,${ }^{173}$ J. Zabel, ${ }^{173}$ A. Zhang, ${ }^{173}$ A. Bodek, ${ }^{174}$ P. de Barbaro, ${ }^{174}$ R. Demina, ${ }^{174}$ Y.t. Duh, ${ }^{174}$ J. L. Dulemba, ${ }^{174}$ C. Fallon, ${ }^{174}$ T. Ferbel, ${ }^{174}$ M. Galanti, ${ }^{174}$ A. Garcia-Bellido, ${ }^{174}$ J. Han,${ }^{174}$ O. Hindrichs, ${ }^{174}$ A. Khukhunaishvili, ${ }^{174}$ P. Tan, ${ }^{174}$ R. Taus,${ }^{174}$ A. Agapitos, ${ }^{175}$ J. P. Chou, ${ }^{175}$ Y. Gershtein, ${ }^{175}$ E. Halkiadakis, ${ }^{175}$ M. Heindl, ${ }^{175}$ E. Hughes, ${ }^{175}$ S. Kaplan, ${ }^{175}$ R. Kunnawalkam Elayavalli, ${ }^{175}$ S. Kyriacou, ${ }^{175}$ A. Lath, ${ }^{175}$ R. Montalvo, ${ }^{175}$ K. Nash, ${ }^{175}$ M. Osherson, ${ }^{175}$ H. Saka, ${ }^{175}$ S. Salur, ${ }^{175}$ S. Schnetzer, ${ }^{175}$ D. Sheffield, ${ }^{175}$ S. Somalwar, ${ }^{175}$ R. Stone, ${ }^{175}$ S. Thomas, ${ }^{175}$ P. Thomassen, ${ }^{175}$ M. Walker, ${ }^{175}$ A. G. Delannoy, ${ }^{176}$ J. Heideman, ${ }^{176}$ G. Riley ${ }_{176}^{176}$ S. Spanier, ${ }^{176}$ O. Bouhali ${ }^{177, \text { www }}$ A. Celik,${ }^{177}$ M. Dalchenko, ${ }^{177}$ M. De Mattia, ${ }^{177}$ A. Delgado, ${ }^{177}$ S. Dildick, ${ }^{177}$ R. Eusebi, ${ }^{177}$ J. Gilmore, ${ }^{177}$ T. Huang, ${ }^{177}$ T. Kamon, ${ }^{177, x x x}$ S. Luo, ${ }^{177}$ R. Mueller, ${ }^{177}$ A. Perloff, ${ }^{177}$ L. Perniè, ${ }^{177}$ D. Rathjens, ${ }^{177}$ A. Safonov, ${ }^{177}$ N. Akchurin, ${ }^{178}$ J. Damgov ${ }^{178}$ F. De Guio, ${ }^{178}$ P. R. Dudero, ${ }^{178}$ S. Kunori,,${ }^{178}$

K. Lamichhane, ${ }^{178}$ S. W. Lee, ${ }^{178}$ T. Mengke, ${ }^{178}$ S. Muthumuni, ${ }^{178}$ T. Peltola, ${ }^{178}$ S. Undleeb,${ }^{178}$ I. Volobouev, ${ }^{178}$ Z. Wang, ${ }^{178}$ S. Greene, ${ }^{179}$ A. Gurrola, ${ }^{179}$ R. Janjam, ${ }^{179}$ W. Johns, ${ }^{179}$ C. Maguire, ${ }^{179}$ A. Melo, ${ }^{179} \mathrm{H}$. Ni, ${ }^{179}$ K. Padeken, ${ }^{179}$ J. D. Ruiz Alvarez, ${ }^{179}$ P. Sheldon, ${ }^{179}$ S. Tuo, ${ }^{179}$ J. Velkovska, ${ }^{179}$ M. Verweij ${ }^{179}$ Q. Xu, ${ }^{179}$ M. W. Arenton, ${ }^{180}$ P. Barria, ${ }^{180}$ B. Cox ${ }^{180}$ R. Hirosky, ${ }^{180}$ M. Joyce, ${ }^{180}$ A. Ledovskoy, ${ }^{180} \mathrm{H}$. Li ${ }^{180}$ C. Neu, ${ }^{180}$ T. Sinthuprasith, ${ }^{180}$ Y. Wang, ${ }^{180}$ E. Wolfe, ${ }^{180}$ F. Xia, ${ }^{180}$ R. Harr, ${ }^{181}$ P. E. Karchin, ${ }^{181}$ N. Poudyal, ${ }^{181}$ J. Sturdy, ${ }^{181}$ P. Thapa, ${ }^{181}$ S. Zaleski, ${ }^{181}$ M. Brodski, ${ }^{182}$ J. Buchanan, ${ }^{182}$ C. Caillol, ${ }^{182}$ D. Carlsmith, ${ }^{182}$ S. Dasu, ${ }^{182}$ L. Dodd, ${ }^{182}$ B. Gomber, ${ }^{182}$ M. Grothe, ${ }^{182}$ M. Herndon, ${ }^{182}$ A. Hervé, ${ }^{182}$ U. Hussain, ${ }^{182}$ P. Klabbers, ${ }^{182}$ A. Lanaro, ${ }^{182}$ K. Long, ${ }^{182}$ R. Loveless, ${ }^{182}$ T. Ruggles, ${ }^{182}$ A. Savin, ${ }^{182}$ V. Sharma, ${ }^{182}$ N. Smith, ${ }^{182}$ W. H. Smith, ${ }^{182}$ and N. Woods ${ }^{182}$

(CMS Collaboration)

\author{
${ }^{1}$ Yerevan Physics Institute, Yerevan, Armenia \\ ${ }^{2}$ Institut für Hochenergiephysik, Wien, Austria \\ ${ }^{3}$ Institute for Nuclear Problems, Minsk, Belarus \\ ${ }^{4}$ Universiteit Antwerpen, Antwerpen, Belgium \\ ${ }^{5}$ Vrije Universiteit Brussel, Brussel, Belgium \\ ${ }^{6}$ Université Libre de Bruxelles, Bruxelles, Belgium \\ ${ }^{7}$ Ghent University, Ghent, Belgium \\ ${ }^{8}$ Université Catholique de Louvain, Louvain-la-Neuve, Belgium \\ ${ }^{9}$ Centro Brasileiro de Pesquisas Fisicas, Rio de Janeiro, Brazil \\ ${ }^{10}$ Universidade do Estado do Rio de Janeiro, Rio de Janeiro, Brazil \\ ${ }^{11 a}$ Universidade Estadual Paulista, São Paulo, Brazil \\ ${ }^{11 \mathrm{~b}}$ Universidade Federal do ABC, São Paulo, Brazil \\ ${ }^{12}$ Institute for Nuclear Research and Nuclear Energy, Bulgarian Academy of Sciences, Sofia, Bulgaria \\ ${ }^{13}$ University of Sofia, Sofia, Bulgaria \\ ${ }^{14}$ Beihang University, Beijing, China \\ ${ }^{15}$ Institute of High Energy Physics, Beijing, China \\ ${ }^{16}$ State Key Laboratory of Nuclear Physics and Technology, Peking University, Beijing, China \\ ${ }^{17}$ Tsinghua University, Beijing, China \\ ${ }^{18}$ Universidad de Los Andes, Bogota, Colombia \\ ${ }^{19}$ University of Split, Faculty of Electrical Engineering, Mechanical Engineering and Naval Architecture, Split, Croatia \\ ${ }^{20}$ University of Split, Faculty of Science, Split, Croatia \\ ${ }^{21}$ Institute Rudjer Boskovic, Zagreb, Croatia \\ ${ }^{22}$ University of Cyprus, Nicosia, Cyprus \\ ${ }^{23}$ Charles University, Prague, Czech Republic \\ ${ }^{24}$ Escuela Politecnica Nacional, Quito, Ecuador \\ ${ }^{25}$ Universidad San Francisco de Quito, Quito, Ecuador \\ ${ }^{26}$ Academy of Scientific Research and Technology of the Arab Republic of Egypt, \\ Egyptian Network of High Energy Physics, Cairo, Egypt \\ ${ }^{27}$ National Institute of Chemical Physics and Biophysics, Tallinn, Estonia \\ ${ }^{28}$ Department of Physics, University of Helsinki, Helsinki, Finland \\ ${ }^{29}$ Helsinki Institute of Physics, Helsinki, Finland
}


${ }^{30}$ Lappeenranta University of Technology, Lappeenranta, Finland

${ }^{31}$ IRFU, CEA, Université Paris-Saclay, Gif-sur-Yvette, France

${ }^{32}$ Laboratoire Leprince-Ringuet, Ecole polytechnique, CNRS/IN2P3, Université Paris-Saclay, Palaiseau, France

${ }^{33}$ Université de Strasbourg, CNRS, IPHC UMR 7178, Strasbourg, France

${ }^{34}$ Centre de Calcul de l'Institut National de Physique Nucleaire et de Physique des Particules, CNRS/IN2P3, Villeurbanne, France

${ }^{35}$ Université de Lyon, Université Claude Bernard Lyon 1, CNRS-IN2P3, Institut de Physique Nucléaire de Lyon, Villeurbanne, France

${ }^{36}$ Georgian Technical University, Tbilisi, Georgia

${ }^{37}$ Tbilisi State University, Tbilisi, Georgia

${ }^{38}$ RWTH Aachen University, I. Physikalisches Institut, Aachen, Germany

${ }^{39}$ RWTH Aachen University, III. Physikalisches Institut A, Aachen, Germany

${ }^{40}$ RWTH Aachen University, III. Physikalisches Institut B, Aachen, Germany

${ }^{41}$ Deutsches Elektronen-Synchrotron, Hamburg, Germany

${ }^{42}$ University of Hamburg, Hamburg, Germany

${ }^{43}$ Karlsruher Institut fuer Technology

${ }^{44}$ Institute of Nuclear and Particle Physics (INPP), NCSR Demokritos, Aghia Paraskevi, Greece

${ }^{45}$ National and Kapodistrian University of Athens, Athens, Greece

${ }^{46}$ National Technical University of Athens, Athens, Greece

${ }^{47}$ University of Ioánnina, Ioánnina, Greece

${ }^{48}$ MTA-ELTE Lendület CMS Particle and Nuclear Physics Group, Eötvös Loránd University, Budapest, Hungary

${ }^{49}$ Wigner Research Centre for Physics, Budapest, Hungary

${ }^{50}$ Institute of Nuclear Research ATOMKI, Debrecen, Hungary

${ }^{51}$ Institute of Physics, University of Debrecen, Debrecen, Hungary

${ }^{52}$ Indian Institute of Science (IISc), Bangalore, India

${ }^{53}$ National Institute of Science Education and Research, HBNI, Bhubaneswar, India

${ }^{54}$ Panjab University, Chandigarh, India

${ }^{55}$ University of Delhi, Delhi, India

${ }^{56}$ Saha Institute of Nuclear Physics, HBNI, Kolkata,India

${ }^{57}$ Indian Institute of Technology Madras, Madras, India

${ }^{58}$ Bhabha Atomic Research Centre, Mumbai, India

${ }^{59}$ Tata Institute of Fundamental Research-A, Mumbai, India

${ }^{60}$ Tata Institute of Fundamental Research-B, Mumbai, India

${ }^{61}$ Indian Institute of Science Education and Research (IISER), Pune, India

${ }^{62}$ Institute for Research in Fundamental Sciences (IPM), Tehran, Iran

${ }^{63}$ University College Dublin, Dublin, Ireland

${ }^{64 a}$ INFN Sezione di Bari, Bari, Italy

${ }^{64 \mathrm{~b}}$ Università di Bari, Bari, Italy

${ }^{64 \mathrm{c}}$ Politecnico di Bari, Bari, Italy

${ }^{65 \mathrm{a}}$ INFN Sezione di Bologna, Bologna, Italy

${ }^{65 \mathrm{~b}}$ Università di Bologna, Bologna, Italy

${ }^{66 \mathrm{a}}$ INFN Sezione di Catania, Catania, Italy

${ }^{66 \mathrm{~b}}$ Università di Catania, Catania, Italy

${ }^{67 \mathrm{a}}$ INFN Sezione di Firenze, Firenze, Italy

${ }^{67 \mathrm{~b}}$ Università di Firenze, Firenze, Italy

${ }^{68}$ INFN Laboratori Nazionali di Frascati, Frascati, Italy

${ }^{69 a}$ INFN Sezione di Genova, Genova, Italy

${ }^{69 \mathrm{~b}}$ Università di Genova, Genova, Italy

${ }^{70 a}$ INFN Sezione di Milano-Bicocca, Milano, Italy

${ }^{70 \mathrm{~b}}$ Università di Milano-Bicocca, Milano, Italy

${ }^{71 a}$ INFN Sezione di Napoli, Napoli, Italy

${ }^{71 b}$ Università di Napoli 'Federico II', Napoli, Italy

${ }^{71 c}$ Università della Basilicata, Potenza, Italy

${ }^{71 \mathrm{~d}}$ Università G. Marconi, Roma, Italy

${ }^{72 a}$ INFN Sezione di Padova, Padova, Italy

${ }^{72 \mathrm{~b}}$ Università di Padova, Padova, Italy

${ }^{72 c}$ Università di Trento, Trento, Italy

${ }^{73 a}$ INFN Sezione di Pavia

${ }^{73 \mathrm{~b}}$ Università di Pavia

${ }^{74 a}$ INFN Sezione di Perugia, Perugia, Italy

${ }^{74 \mathrm{~b}}$ Università di Perugia, Perugia, Italy

${ }^{75 a}$ INFN Sezione di Pisa, Pisa, Italy 


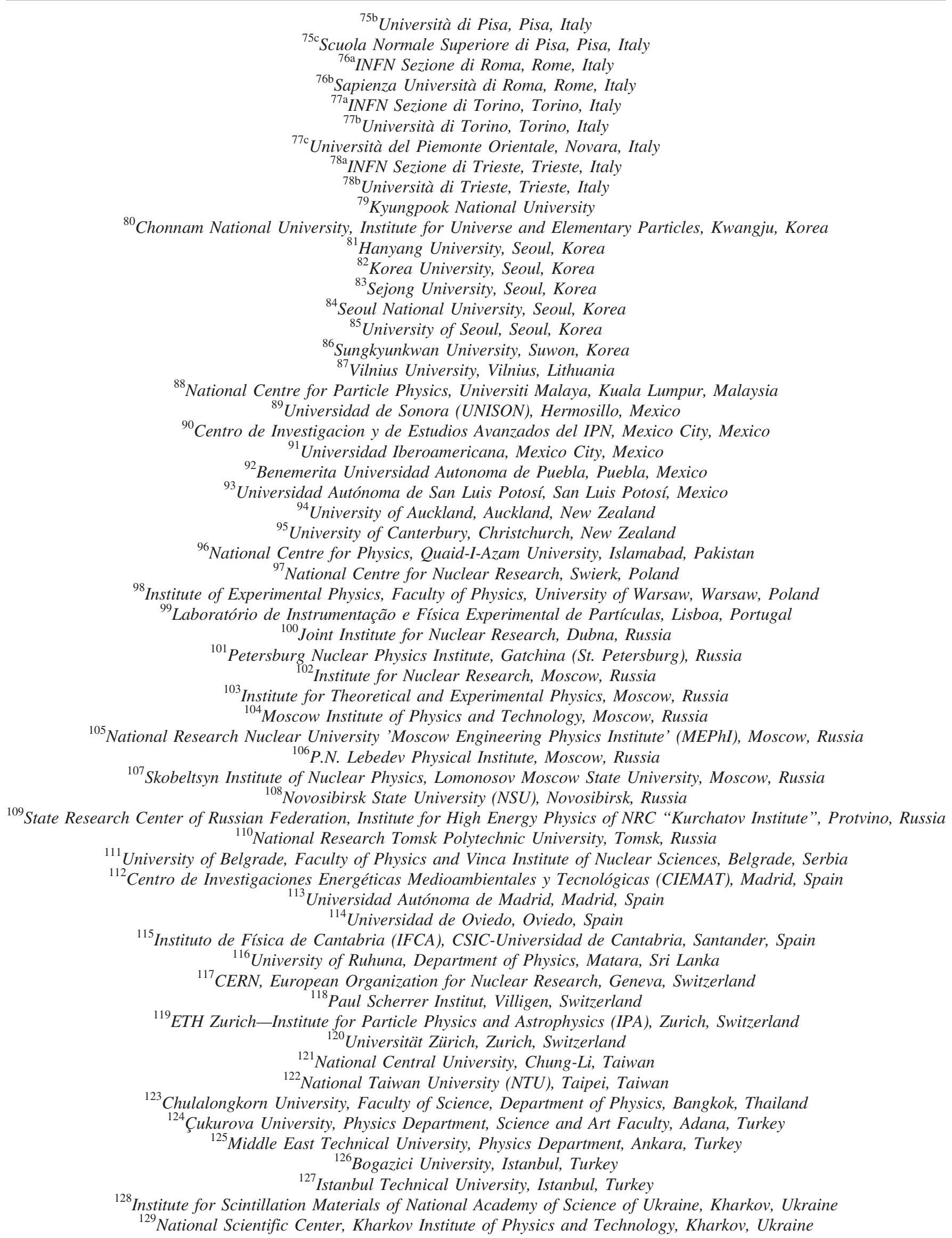


PHYSICAL REVIEW LETTERS 121, 221802 (2018)

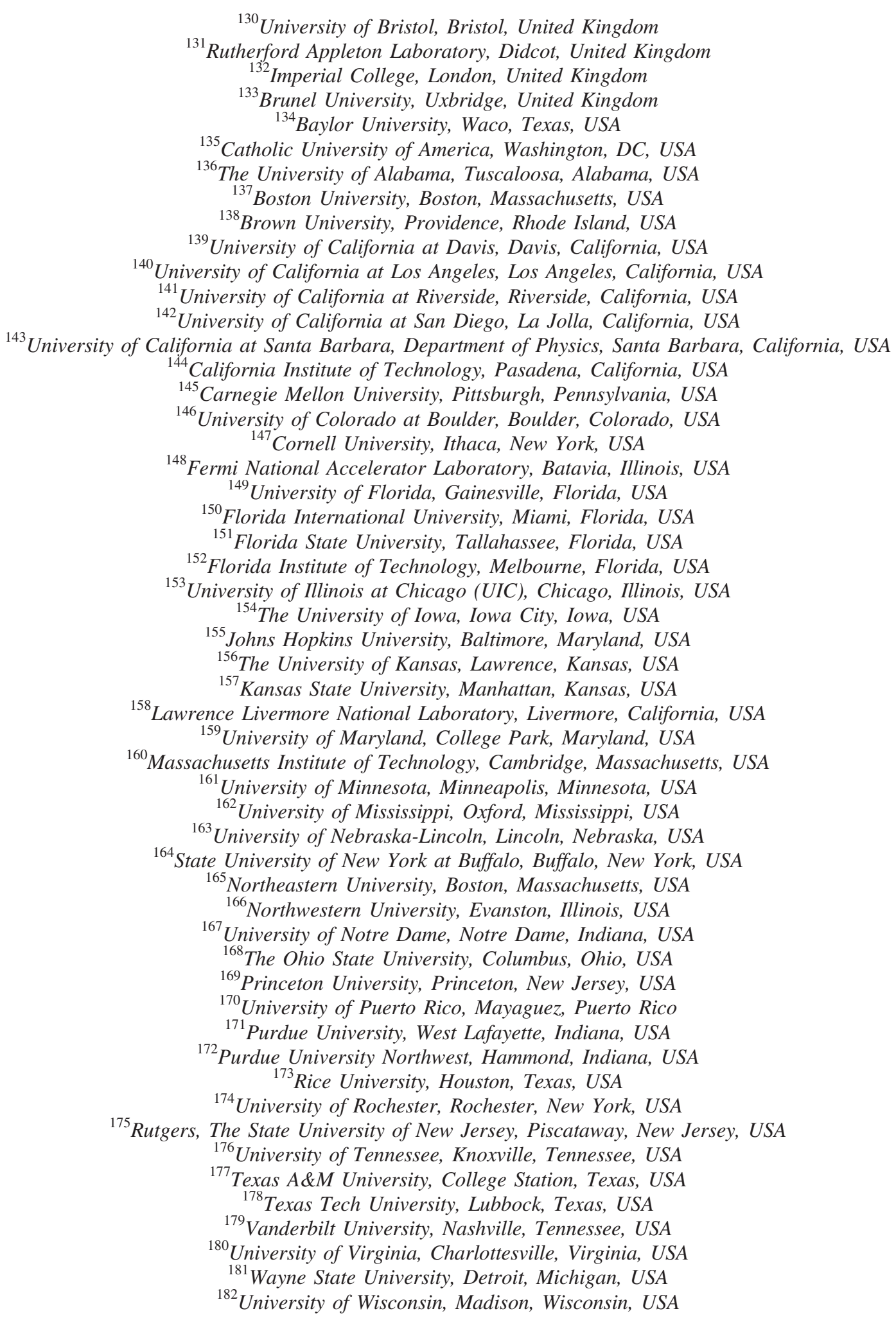

${ }^{\mathrm{a}}$ Deceased.

${ }^{\mathrm{b}}$ Also at Vienna University of Technology, Vienna, Austria.

${ }^{\mathrm{c}}$ Also at IRFU, CEA, Université Paris-Saclay, Gif-sur-Yvette, France.

${ }^{\mathrm{d}}$ Also at Universidade Estadual de Campinas, Campinas, Brazil.

${ }^{\mathrm{e}}$ Also at Federal University of Rio Grande do Sul, Porto Alegre, Brazil.

${ }^{\mathrm{f}}$ Also at Université Libre de Bruxelles, Bruxelles, Belgium. 
${ }^{g}$ Also at University of Chinese Academy of Sciences, Beijing, China.

${ }^{\mathrm{h}}$ Also at Institute for Theoretical and Experimental Physics, Moscow, Russia.

${ }^{\mathrm{i}}$ Also at Joint Institute for Nuclear Research, Dubna, Russia.

${ }^{\mathrm{j}}$ Also at Cairo University, Cairo, Egypt.

${ }^{k}$ Also at Helwan University, Cairo, Egypt.

${ }^{1}$ Also at Zewail City of Science and Technology, Zewail, Egypt.

${ }^{\mathrm{m}}$ Also at Ain Shams University, Cairo, Egypt.

${ }^{\mathrm{n}}$ Also at British University in Egypt, Cairo, Egypt.

${ }^{\circ}$ Also at Department of Physics, King Abdulaziz University, Jeddah, Saudi Arabia.

${ }^{\mathrm{p}}$ Also at Université de Haute Alsace, Mulhouse, France.

${ }^{\mathrm{q}}$ Also at Skobeltsyn Institute of Nuclear Physics, Lomonosov Moscow State University, Moscow, Russia.

${ }^{\mathrm{r}}$ Also at CERN, European Organization for Nuclear Research, Geneva, Switzerland.

${ }^{\text {s} A l s o ~ a t ~ R W T H ~ A a c h e n ~ U n i v e r s i t y, ~ I I I . ~ P h y s i k a l i s c h e s ~ I n s t i t u t ~ A, ~ A a c h e n, ~ G e r m a n y . ~}$

${ }^{\mathrm{t}}$ Also at University of Hamburg, Hamburg, Germany.

"Also at Brandenburg University of Technology, Cottbus, Germany.

${ }^{v}$ Also at MTA-ELTE Lendület CMS Particle and Nuclear Physics Group, Eötvös Loránd University, Budapest, Hungary.

${ }^{w}$ Also at Institute of Nuclear Research ATOMKI, Debrecen, Hungary.

${ }^{\mathrm{x}}$ Also at Institute of Physics, University of Debrecen, Debrecen, Hungary.

${ }^{\mathrm{y}}$ Also at IIT Bhubaneswar, Bhubaneswar, India.

${ }^{\mathrm{z}}$ Also at Institute of Physics, Bhubaneswar, India.

${ }^{a a}$ Also at Shoolini University, Solan, India.

${ }^{\text {bb }}$ Also at University of Visva-Bharati, Santiniketan, India.

${ }^{c c}$ Also at Isfahan University of Technology, Isfahan, Iran.

${ }^{\mathrm{dd}}$ Also at Plasma Physics Research Center, Science and Research Branch, Islamic Azad University, Tehran, Iran.

${ }^{e e}$ Also at Università degli Studi di Siena, Siena, Italy.

${ }^{\mathrm{ff}}$ Also at Kyunghee University, Seoul, Korea.

${ }^{g g}$ Also at International Islamic University of Malaysia, Kuala Lumpur, Malaysia.

${ }^{\text {hh }}$ Also at Malaysian Nuclear Agency, MOSTI, Kajang, Malaysia.

${ }^{i i}$ Also at Consejo Nacional de Ciencia y Tecnología, Mexico City, Mexico.

${ }^{\mathrm{jj}}$ Also at Warsaw University of Technology, Institute of Electronic Systems, Warsaw, Poland.

${ }^{\mathrm{kk}}$ Also at Institute for Nuclear Research, Moscow, Russia.

"Also at National Research Nuclear University 'Moscow Engineering Physics Institute' (MEPhI), Moscow, Russia.

${ }^{\mathrm{mm}}$ Also at St. Petersburg State Polytechnical University, St. Petersburg, Russia.

${ }^{\mathrm{nn}}$ Also at University of Florida, Gainesville, Florida, USA.

${ }^{00}$ Also at P.N. Lebedev Physical Institute, Moscow, Russia.

${ }^{\mathrm{pp}}$ Also at California Institute of Technology, Pasadena, California, USA.

${ }^{\mathrm{qq}}$ Also at Budker Institute of Nuclear Physics, Novosibirsk, Russia.

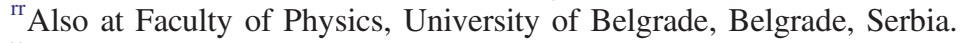

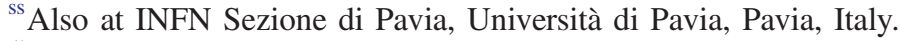

"Also at University of Belgrade, Faculty of Physics and Vinca Institute of Nuclear Sciences, Belgrade, Serbia.

${ }^{\text {uu } A l s o ~ a t ~ S c u o l a ~ N o r m a l e ~ e ~ S e z i o n e ~ d e l l ' I N F N, ~ P i s a, ~ I t a l y . ~}$

${ }^{\mathrm{vv}}$ Also at National and Kapodistrian University of Athens, Athens, Greece.

${ }^{w w}$ Also at Riga Technical University, Riga, Latvia.

${ }^{\mathrm{xx}}$ Also at Universität Zürich, Zurich, Switzerland.

${ }^{y y}$ Also at Stefan Meyer Institute for Subatomic Physics, Vienna, Austria.

${ }^{\mathrm{zz}}$ Also at Adiyaman University, Adiyaman, Turkey.

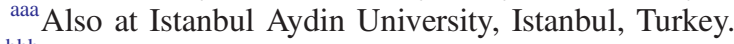

${ }^{\text {bbb }}$ Also at Mersin University, Mersin, Turkey.

${ }^{c c c}$ Also at Piri Reis University, Istanbul, Turkey.

${ }^{\text {ddd }}$ Also at Gaziosmanpasa University, Tokat, Turkey.

${ }^{\text {eee }}$ Also at Ozyegin University, Istanbul, Turkey.

${ }^{\text {fff }}$ Also at Izmir Institute of Technology, Izmir, Turkey.

${ }^{\text {ggg }}$ Also at Marmara University, Istanbul, Turkey.

hhh Also at Kafkas University, Kars, Turkey.

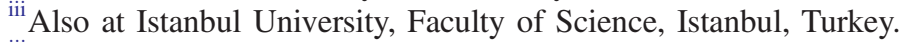

${ }^{i j j}$ Also at Istanbul Bilgi University, Istanbul, Turkey.

${ }^{\mathrm{kkk}}$ Also at Hacettepe University, Ankara, Turkey.

${ }^{111}$ Also at Rutherford Appleton Laboratory, Didcot, United Kingdom.

${ }^{\mathrm{mmm}}$ Also at School of Physics and Astronomy, University of Southampton, Southampton, United Kingdom.

${ }^{\mathrm{nnn}}$ Also at Monash University, Faculty of Science, Clayton, Australia. 


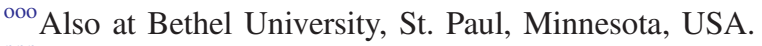

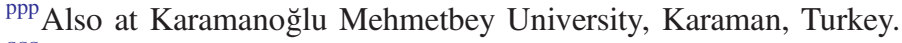

${ }^{\mathrm{qqq}}$ Also at Utah Valley University, Orem, Utah, USA.

${ }^{\mathrm{rrr}}$ Also at Purdue University, West Lafayette, Indiana, USA.

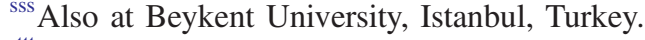

${ }^{\mathrm{ttt}}$ Also at Bingol University, Bingol, Turkey.

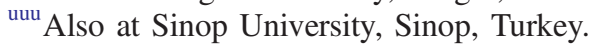

${ }^{\mathrm{vvv}}$ Also at Mimar Sinan University, Istanbul, Istanbul, Turkey.

${ }^{w w w}$ Also at Texas A\&M University at Qatar, Doha, Qatar.

${ }^{\mathrm{xxx}}$ Also at Kyungpook National University, Daegu, Korea.
} 